\title{
Accurate photometry with adaptive optics in the presence of anisoplanatic effects with a sparsely sampled PSF
}

\section{The Galactic center as an example of a challenging target for accurate AO photometry}

\author{
R. Schödel \\ Instituto de Astrofísica de Andalucía (CSIC), C/ Camino Bajo de Huétor 50, 18008 Granada, Spain \\ e-mail: rainer@iaa.es
}

Received 1 July 2009 / Accepted 12 October 2009

\begin{abstract}
Context. Anisoplanatic effects can cause significant systematic photometric uncertainty in the analysis of dense stellar fields observed with adaptive optics. Program packages have been developed for a spatially variable PSF, but they require that a sufficient number of bright, isolated stars in the image are present to adequately sample the PSF.

Aims. Imaging the Galactic center is particularly challenging. We present two ways of dealing with spatially variable PSFs when only one or very few suitable PSF reference stars are present in the field.

Methods. Local PSF fitting with the StarFinder algorithm is applied to the data. Satisfying results can be found in two ways: (a) creating local PSFs by merging locally extracted PSF cores with the PSF wings estimated from the brightest star in the field; (b) Wiener deconvolution of the image with the PSF estimated from the brightest star in the field and subsequent estimation of local PSFs on the deconvolved image. The methodology is tested on real, and on artificial images.

Results. The method involving Wiener deconvolution of the image prior to local PSF extraction and fitting gives excellent results. It limits systematic effects to $\sim 2-5 \%$ in point source photometry and $\sim 10 \%$ in diffuse emission on fields-of-view as large as $28^{\prime \prime} \times 28^{\prime \prime}$ and observed through the $H$-band filter. Particular attention is given to how deconvolution changes the noise properties of the image. It is shown that mean positions and fluxes of the stars are conserved by the deconvolution. However, the estimated uncertainties of the PSF fitting algorithm are too small if the presence of covariances is ignored in the PSF fitting as has been done here. An appropriate scaling factor can, however, be determined from simulated images or by comparing measurements on independent data sets.

Conclusions. We present ways of obtaining reliable photometry and astrometry from images with a spatially variable, but poorly sampled PSF, where standard techniques may not work.
\end{abstract}

Key words. techniques: image processing - instrumentation: high angular resolution - instrumentation: adaptive optics methods: data analysis - methods: observational - Galaxy: center

\section{Introduction}

Aperture photometry is clearly the most straightforward and when examining isolated sources - most accurate means of determining photometry. Nevertheless, it runs into serious difficulty in crowded fields. Frequently, adaptive optics (AO) is applied to near-infrared observations of crowded stellar fields. This reduces source confusion considerably, but the limited Strehl ratio leads to extended wings of the point spread function (PSF). As a result, the complete PSF usually has a diameter of the order of the seeing disk, even if a large fraction of the flux is concentrated inside a circular region with a radius of at most a few times the diffraction limit. Therefore, the light from stars in dense fields will overlap even when using AO techniques. For this reason, crowded stellar fields are usually analyzed with PSF fitting program packages, such as DAOPHOT (Stetson 1987) or StarFinder (Diolaiti et al. 2000b). The latter program package was written explicitly for use with AO data and uses an empirical PSF that is extracted directly from the data.

If the PSF is constant over the field, the astrometric and photometric accuracy of PSF fitting algorithms is limited only by the signal-to-noise ratio of the imaging data and the accuracy of one's knowledge of the PSF. However, the PSF is never constant across the field-of-view (FOV), but subject to changes caused by distortions and aberrations in the optical path. A particularly strong effect is the change of the PSF as a function of distance from the guide star in AO observations. This effect, termed anisoplanacy, is due to different lines-of-sight probing different parts of the turbulent atmosphere. Various methods have been developed for taking anisoplanatic effects into account a posteriori. Some of these methods involve, e.g., source fitting with local PSF estimates on subfields smaller than the isoplanatic angle (e.g., Diolaiti et al. 2000b; Christou et al. 2004), space-variant deconvolution (e.g., Diolaiti et al. 2000b), analytical formulations of anisoplanatism combined with the guide star PSF and information about the atmospheric turbulence profile (Britton 2006), or semi-empirical PSF modeling based on observations of dense stellar fields (Steinbring et al. 2002). The PSF fitting program package DAOPHOT deals with variable PSFs by allowing for up to a quadratic variability in mathematical PSF models and combining those with local look-up tables (e.g., Stetson 1992). Multiconjugate (MCAO, Beckers 1988) or multiobject AO (Hammer et al. 2004; Ellerbroek et al. 2005) techniques will reduce the effects of anisoplanatism a priori in future 
observations, i.e., before the light reaches the detector. The multiconjugate AO technique has already been successfully demonstrated on the ESO VLT using the Multiconjugate Adaptive Optics Demonstrator (e.g., Bouy et al. 2008; Gullieuszik et al. 2008; Marchetti et al. 2008). Nevertheless, while MCAO improves the Strehl ratio of sources dramatically over a wide FOV, significant and difficult to predict PSF variations across the FOV remain. Dealing with spatially variable PSFs in photometry is therefore important and will remain so in the near future.

All approaches to PSF fitting with spatially variable PSFs need a sufficient number of bright, isolated PSF reference stars that are approximately homogeneously distributed across the FOV. In other words, the PSF and its spatial variation must be adequately sampled. In this paper, I present a data set that poses particular problems for this latter assumption. Two ways are proposed on how to overcome the difficulties. The most successful method consists of a combination of linear, Wiener-filter deconvolution and local PSF fitting. The image is first Wiener-filterdeconvolved using a suitable PSF, ideally the one of the guiding star, to reduce crowding. In a second step, the local variation in the PSF and the ringing introduced by the Wiener filter technique is taken care of by PSF fitting with locally extracted PSFs. The method is easy to implement. Deconvolution will lead to covariances in the noise. It is shown that this does not lead to erroneous measurements for Wiener deconvolution and the imaging data used here. However, care must be taken to obtain accurate estimates of the uncertainties in the positions and fluxes.

The algorithm was developed and tested on near-infrared observations of the crowded Galactic center field with NACO at the ESO VLT and should be applicable in general to AO observations of crowded fields. An important prerequisite however, is a sufficient density of point sources over the entire FOV to be able to estimate the PSF after deconvolution with sufficient and nearly constant quality across the field. Applied to the data analyzed in this work, systematic variations of point source photometry out to distances of $\sim 30^{\prime \prime}$ from the guide star can be limited to $\$ 2-5 \%$. Additionally, the method presented does not only lead to accurate point source photometry, but also allows one to determine the diffuse emission due to unresolved stars with a $1 \sigma$ accuracy of $\sim 0.1 \mathrm{mag} \operatorname{arcsec}^{-2}$ across the entire FOV.

\section{Data}

The observations used in this work were obtained with the nearinfrared camera and AO system NAOS/CONICA (short NACO) at the ESO VLT unit telescope $4^{1}$. The $\operatorname{mag}_{K \mathrm{~s}} \approx 6.5-7.0 \mathrm{su}-$ pergiant IRS 7 was used to close the loop of the AO, using the instrument's unique NIR wavefront sensor. The sky background was measured on a largely empty patch of sky, a dark cloud about 400" north and 713" east of the target. Sky subtraction, bad pixel correction, and flat fielding were applied to the individual exposures. The NACO S27 camera, with a pixel scale of $0.027^{\prime \prime} /$ pix, was used for the $H$-band observations. The field-ofview (FOV) of a single exposure is thus $28^{\prime \prime} \times 28^{\prime \prime}$. The observations were dithered by applying a rectangular dither pattern with the center of the dithered exposures positioned approximately at $\left(8.0^{\prime \prime},-2.6^{\prime \prime}\right),\left(-6.1^{\prime \prime},-2.7^{\prime \prime}\right),\left(-6.1^{\prime \prime}, 11.2^{\prime \prime}\right)$, and $\left(8.1^{\prime \prime}, 11.3^{\prime \prime}\right)$ east and north of $\mathrm{Sgr}^{*}$. In the text we refer to these four offsets as dither positions $1,2,3$, and 4 . The combined FOV of the observations is about $40^{\prime \prime} \times 40^{\prime \prime}$ and is offset to the north with

\footnotetext{
1 Based on observations made with ESO Telescopes at the La Silla or Paranal Observatories under programme ID 077.B-0014.
}

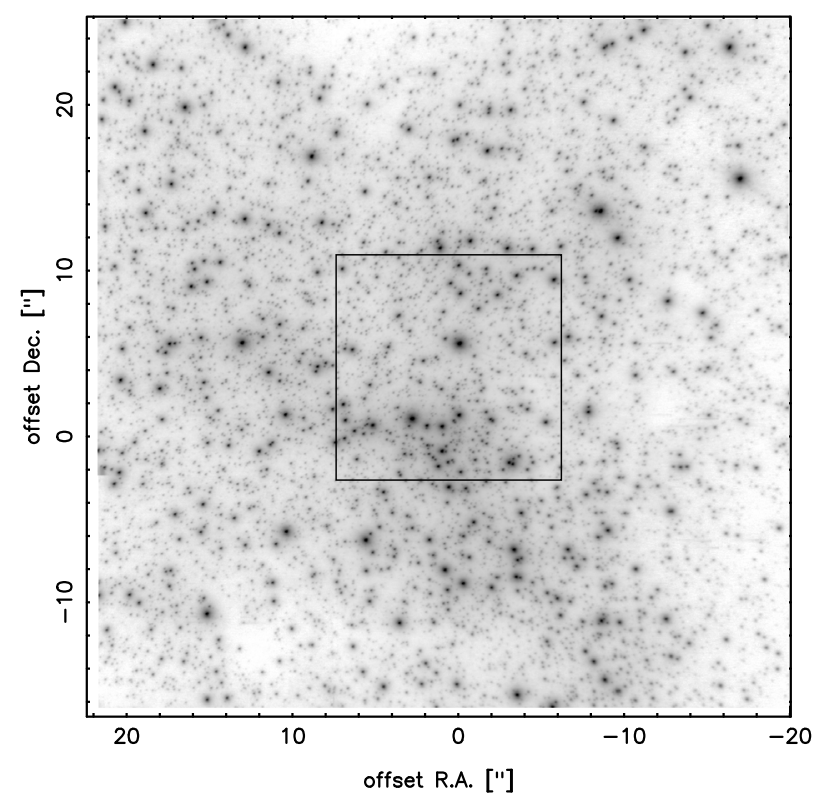

Fig. 1. Mosaic image of the $H$-band observations from 29 April 2006. North is up and east is to the left. Offsets are given in arcseconds from Sgr A*. The black rectangle indicates the area of overlap between the 4 dither positions.

Table 1. Details of the imaging observations used in this work.

\begin{tabular}{llllll}
\hline \hline Date & $\begin{array}{l}\lambda_{\text {central }} \\
{[\mu \mathrm{m}]}\end{array}$ & $\begin{array}{l}\Delta \lambda \\
{[\mu \mathrm{m}]}\end{array}$ & $N^{a}$ & NDIT $^{b}$ & $\begin{array}{l}\mathrm{DIT}^{c} \\
{[\mathrm{~s}]}\end{array}$ \\
\hline 29 April 2006 & 1.66 & 0.33 & 32 & 28 & 2 \\
\hline
\end{tabular}

${ }^{a}$ Number of (dithered) exposures ${ }^{b}$ Number of integrations that were averaged on-line by the read-out electronics ${ }^{c}$ Detector integration time. The total integration time of each observation amounts to $N \times$ NDIT $\times$ DIT.

respect to $\mathrm{Sgr} \mathrm{A}^{*}$ because the guide star IRS 7 is located about 5.6" north of Sgr A*.

Seeing ranged between $0.6^{\prime \prime}$ and $1.0^{\prime \prime}$. The Strehl ratio that was achieved ranged between $\sim 15 \%$ near the guide star and $\sim 8 \%$ at $25^{\prime \prime}$ distance from the guide star. The Strehl ratio was estimated using the Strehl algorithm of the ESO eclipse software package (Devillard 1997) on PSFs extracted at various positions in the image. From the multiple measurements, we estimated the $1 \sigma$ uncertainty of the measured Strehl ratio to be $\sim 3 \%$. Sky transparency variations were below $1 \%$ during the observations. Table 1 summarizes the observations. The detector integration time (DIT) was set to be $2.0 \mathrm{~s}$ to avoid saturation of the brightest stars. After 28 DITs, the instrument averaged the data to a single exposure (NDIT $=28)$. In this way, 8 individual exposures were obtained per dither position. The exposures of each respective dither position were aligned (to compensate for small residual shifts) with the jitter algorithm of the ESO eclipse software package. We show the combined FOV of the $H$-band observations in Fig. 1. We note that the photometry and astrometry were performed on the combined images of each dither position and not on the combined mosaic of all images (as shown in Fig. 1) to maintain a constant signal-to-noise ratio over the entire images. The $\sim 13.5^{\prime \prime} \times 13.5^{\prime \prime}$ overlap area between the four dither positions is indicated by the rectangle in Fig. 1.

Zero points for the NACO instrument for various combinations of cameras, filters, and dichroics (that divide the light between wavefront sensor and camera) are determined routinely 
within the ESO instrument calibration plan. The zero point for the $H$-band and for the corresponding setup (camera S27, dichroic N20C80) was determined by observing a standard star during the same night as the observations: $Z P_{H}=23.64 \pm 0.05$.

Anisoplanatic effects are stronger at shorter wavelengths, therefore I chose $H$-band data (instead of $K \mathrm{~s}$, the usual filter for observing the Galactic center because of the strong extinction) to test the photometric methodology presented in this work.

\section{PSF fitting photometry and astrometry}

Photometry and astrometry in crowded fields is usually performed with a PSF fitting algorithm. Perhaps the most widely used software of this kind is DAOPHOT (e.g., Stetson 1987, 1992), which is also integrated in the IRAF software package. Another popular package is SExtractor (Bertin \& Arnouts 1996). In this work, I use the StarFinder algorithm (Diolaiti et al. 2000a), which was specifically developed for images obtained by AO assisted observations and is fairly popular in the AO community. It has been shown to obtain comparable results to those of DAOPHOT (in the isoplanatic case, see Diolaiti et al. 2000a,b). I also experimented with DAOPHOT (see Sect. 5.2).

In the StarFinder algorithm, an empirical point spread function (PSF) is extracted by using one or several stars in the image. Cross correlation on potential stars is performed. A correlation threshold is set to accept/reject potential stars. Gaussian readout noise and Poissonian photon noise are determined by the algorithm and taken into account in the fitting process to determine formal uncertainties. A smooth diffuse background emission is fit for the image simultaneously to the point source photometry and astrometry. The PSF extraction can be iteratively improved by using the measured positions and fluxes of detected stars for removal of secondary sources near the PSF reference stars.

There are many parameters that can be modified in the StarFinder algorithm. The most important ones are the number of iterations and the point source detection threshold applied at each iteration, the size of the box for background estimation, and the correlation threshold. The parameter thresh gives both the number of iterations and the threshold in terms of standard deviations from the noise, e.g., thresh $=[3 ., 3$.] means two iterations with a $3 \sigma$ threshold for each one. The parameter back_box is given in pixels and designates a box size. StarFinder estimates the sky background in boxes of size back_box $\times$ back_box and then computes a smooth background by means of interpolation between the background grid points. By default, StarFinder applies bilinear interpolation. We found that this tends to overestimate the background close to bright point sources and therefore chose cubic interpolation. The minimum required value for a correlation to exist between a point source and the PSF is given by min_corr. In this work, the following values of these parameters were applied: thresh $=$ [5.] for a first detection of sources that are subsequently used to iteratively improve the PSF; thresh $=[5 ., 5$.] for point source extraction; back_box $=60$ (back_box $=30$ on deconvolved images); and min_corr $=0.8$ (min_corr $=0.9$ on deconvolved images).

For accurate photometry and astrometry, a number of effects must be taken into account in addition to this basic methodology. We identified the following points as being particularly important:

- accuracy of the empirically determined PSF;

- variation in the PSF across the field;

- influence of deconvolution techniques on the results.
The last point is in part a consequence of the first two points and the desire to improve the accuracy of the applied methodology. In the following subsections different aspects of the PSF fitting method are addressed in detail.

In this section, we address the points raised in the above list. We use $H$-band imaging data in our analysis of anisoplanatic effects because the variation in the PSF across the field is normally stronger at shorter wavelengths. Some concern may be raised because the ideal diffraction-limited PSF of the VLT in the $H$-band is barely Nyquist sampled with the $0.027^{\prime \prime}$ per pixel camera scale. However, the FWHM of the PSFs over the entire FOV is $>3$ pixels in all the images. This means that the PSF is sufficiently well sampled and the fitting algorithm applied by StarFinder works reliably, as we have also been able to confirm with simulated images (see Sect. 4).

\subsection{PSF extraction}

The StarFinder algorithm uses of an empirical PSF, i.e., a PSF directly extracted from the imaging data. This is recommendable in AO observations because of the complexity of the PSF (e.g., a partial, possibly broken, airy pattern superposed on a Gaussian seeing disk, and speckles in the PSF wings) that cannot be easily described by mathematical models. The PSF in StarFinder is determined from the median superposition of various stars or, alternatively, from a single, bright, isolated, and unsaturated star. Knowledge of the empirical PSF is limited because of the limited $\mathrm{S} / \mathrm{N}$ of the imaging data and the presence of secondary sources in the wings of the PSFs of the reference stars. Radial and angular smoothing of the wings of the empirically determined PSF is implemented to improve the $\mathrm{S} / \mathrm{N}$ in the faint wings.

The optimal PSF reference star is as bright as possible without being saturated and completely isolated, i.e., with no secondary sources close to the core or in the seeing wings. These requirements are not easy to fulfill in crowded fields. In the case of the Galactic center (GC), the supergiant IRS 7 has been used routinely as a PSF reference star for GC observations with NACO since the year 2002. Interferometric observations with the ESO VLT Interferometer (VLTI) confirm that this supergiant is unresolved at $2 \mu \mathrm{m}$ with an aperture of $8 \mathrm{~m}$ (Pott et al. 2008). In the near-infrared, any star within $0.5^{\prime \prime}$ of IRS 7 is $>4$ mag fainter (even taking into account the variability of IRS 7; see Blum et al. 1996 and Ott et al. 1999). The brightest star within $1^{\prime \prime}$ of IRS 7 is $>3$ mag fainter than IRS 7. An iterative approach is implemented in the StarFinder code that helps us to effectively remove secondary sources in the PSF estimation process. IRS 7 is therefore well suited to providing a PSF reference.

In the left panel of Fig. 2, we show the image corresponding to dither position 3 (see Sect. 3). The inset shows the PSF extracted from IRS 7. Besides the iterative approach, the $\mathrm{S} / \mathrm{N}$ in the faint wings was improved by applying the HALO_SMOOTH routine provided by StarFinder (angular width set to $180 \mathrm{deg}$, radial width to 20 pixels). As can be seen, the PSF is clearly defined out to distances $>1^{\prime \prime}$ from the PSF core. The right panel of Fig. 2 shows the residuals ${ }^{2}$ across the image that result after fitting the point sources and the diffuse emission in the image using the PSF extracted from IRS 7. For clearer illustration, the linear gray scale of the image of the residuals has been clipped. As can

\footnotetext{
${ }^{2}$ In this work, residuals are defined as image - point sources smooth diffuse emission, such as given by StarFinder. This means that the residual image should ideally fluctuate around zero. Negative (positive) residuals around bright stars will have equivalent positive (negative) regions of diffuse background emission associated with them.
} 

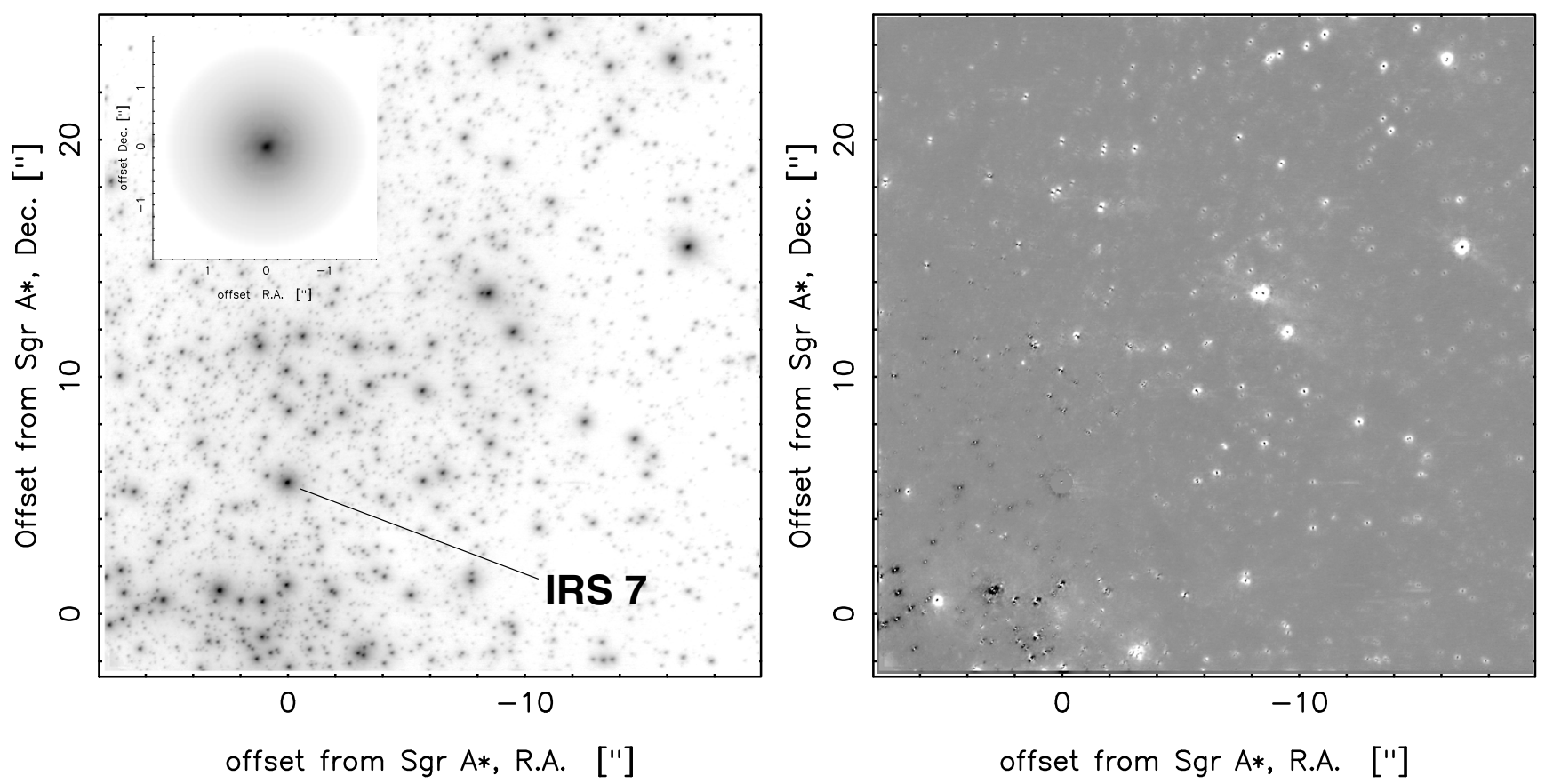

Fig. 2. Left: image from dither position 3 (see Sect. 3). The gray scale is logarithmic. The position of IRS 7, the guide star, and the PSF reference, is indicated. The inset shows the PSF extracted from IRS 7 on a logarithmic gray scale. Right: residuals (linear gray scale) after analyzing the image with the PSF extracted from IRS 7. Here, the gray scale is linear and has been clipped for clearer illustration.

be seen, the residuals related to point sources vary systematically across the FOV. This demonstrates how using a single, fixed PSF leads inevitably to systematic errors in the photometry when the FOV is larger than the isoplanatic angle.

\subsection{Local extraction of the PSF}

The FOV of the NACO S27 camera $\left(28^{\prime \prime} \times 28^{\prime \prime}\right)$ is larger than the isoplanatic angle, which is of the order $\sim 10^{\prime \prime}$ in the $H$-band, but depending strongly on the momentary atmospheric conditions. This leads to a drop in the Strehl ratio and a change of the shape of the PSF with distance from the guide star. Usually the PSF appears elongated, the long axis pointing toward the guide star. By using a single PSF in the analysis of extensive AO observations with a large FOV, systematic errors in both photometry and astrometry over the entire image can therefore be produced (see right panel of Fig. 2). At large distances from the guide star, anisoplanatic effects can even cause the detection of spurious sources because PSF fitting algorithms such as StarFinder may try to separate elongated sources into two or more stars (see Schödel et al. 2007).

An obvious way to take anisoplanatic effects into account is by using locally extracted PSFs. A prerequisite for this technique is a sufficiently high density of point sources across the FOV. This condition is clearly fulfilled in the GC observations analyzed here. However, while there are many point sources all over the field, there exist large patches of the FOV, where there are only faint stars. An additional difficulty is that the radius of the PSF seeing foot, as determined from the guide star, is very large, $\sim 60$ pixels or $1.62^{\prime \prime}$. Therefore, it is all but impossible to find bright, isolated stars homogeneously distributed over the field that could adequately sample the local PSF. Nevertheless, as shown in the section above, a reliable PSF, including its faint, very extended wings, can be extracted from the brightest star in the field, IRS 7, by applying an iterative approach. Anisoplanatic effects are caused by the AO correction being optimized within the isoplanatic patch. The seeing foot in AO PSFs is caused by the uncorrected light.

Anisoplanatic effects will be much stronger in the core of the PSF than in the seeing foot. We illustrate this in Fig. 5, where we show the PSF at the position of the guide star and the PSF at a distance greater than the isoplanatic angle ${ }^{3}$. As can be seen in the difference image at the right panel in Fig. 5, the difference between the two PSFs is negligible in the wings. Convolution with an elongated kernel of a few pixel size will not have strong effects on features with spatial scales of several tens of pixels.

Consequently, a viable approach to local PSF fitting in an anisoplanatic image with inadequately distributed reference stars, as in the case of the presented data, may be to determine the cores of the PSFs locally and then merge these cores with the accurately determined wings of the PSF from the guide star. To merge the local PSFs with the guide star PSF, I used the StarFinder routine to repair saturated stars. After some experimenting, I decided to limit the locally determined core to 3 times the FWHM of the stars. Of course, the larger the local core size that can be chosen, the better, but there is a trade-off because in some areas of the field only faint $(K \mathrm{~s} \gtrsim 14)$ stars are available for PSF extraction.

Local PSF fitting was performed by dividing the image into rectangular fields smaller than the isoplanatic angle (see also Diolaiti et al. 2000b; Schödel et al. 2007, 2009). The $1024 \times$ 1024 pixel $^{2}$ field field was partitioned into $13 \times 13$ overlapping subimages of $256 \times 256$ pixel $^{2}\left(\sim 6.9^{\prime \prime} \times 6.9^{\prime \prime}\right)$. The shifts between subimages are thus just 64 pixels in the $x$ - and/or $y$-direction and there is large overlap between the subframes. This allows us to measure most stars a multiple number of times with different

\footnotetext{
${ }^{3}$ Note that the latter is actually a model PSF, created by convolution of the guide star PSF with the local kernel that was extracted from the Lucy-Richardson deconvolved image (see Sect. 3.3 and Fig. 6).
} 
sets of PSFs. Of the order 200 PSF reference stars distributed over the full FOV were marked manually (using the StarFinder widget interface) before running the automated analysis of the subframes.

When choosing the size of the subframes there is some tradeoff between having a sufficient number of reference stars in the field for accurate PSF estimation and the requirement to keep the subframe as small as possible to keep anisoplanatic effects to a minimum. The subframe size used in this work was found after experimenting with various sizes. It is difficult to find an objective measure for this quantity. Nevertheless, the experiments showed that there is a significant tolerance in the results in terms of the exact frame-size chosen, which can vary by several arcseconds.

The positions and fluxes of each star as well as the corresponding formal uncertainties were computed by taking the mean of the multiple measurements and the corresponding formal uncertainties from overlapping frames (by formal uncertainty we refer in this work to the uncertainty estimated by the StarFinder algorithm for each fit, based on the given PSF and Gaussian and photon noise). The astrometric and photometric uncertainties caused by the uncertainty in the estimated PSF were estimated from the standard deviation of the multiple measurements from the overlapping frames (We refer to this source of uncertainty in this work as PSF uncertainty.). Since the PSFs of the different subframes are not strictly statistically independent (common PSF reference stars in overlapping subframes), the uncertainty in the mean was computed by dividing the standard deviation by $\sqrt{(N / 3)}$ instead of $\sqrt{(N)}$, where $N$ is the number of measurements for a given star. The factor 3 here is not strictly mathematically derived, but estimated, motivated by the shift between subframes being one quarter of their width. Additionally, stars near the edge of a sub-frame (half a shiftwidth) are excluded from the measurements (except near the edge of the combined FOV) to avoid edge effects. This means that about $1 / 3$ of the PSF reference stars will be different in the shifted frame. No weighting was applied to the PSF reference stars. Experiments with weighting had poorer results. This may be caused by sporadic non-ideal PSF reference stars (e.g., with very close companions). Weighting would also mean that one bright star can dominate entire sub-frames, which counteracts the attempt to sample the PSF as locally as possible and would reduce the independence of the measurements. A comparison between the resulting PSF uncertainties and formal photometric uncertainties is shown in Fig. 3. There are two important observations from the comparison of these two sources of uncertainty: the formal uncertainty is a strong function of magnitude and the PSF uncertainty appears to be constant, which ultimately limits the photometric accuracy for bright stars.

Finally, the residuals (image minus detected-point-sources minus diffuse emission) of the various sub-frames were combined to obtain the residuals of the entire FOV. It is shown in the left panel of Fig. 4. The residuals are reduced significantly compared to the single PSF case. There are some negative residuals, which are especially visible around the brightest sources. The reason for these residuals is the difficulty of local PSF extraction combined with the difficulty of merging the local PSF core with the wings of the PSF extracted from IRS 7. It may be possible to improve this process, but the related systematic errors are not larger than a few percent (see Sect. 4, where this method is tested on a simulated image).

The next sub-section describes a way in which the local PSF fitting can be improved by combining it with linear deconvolution techniques. quadrant3

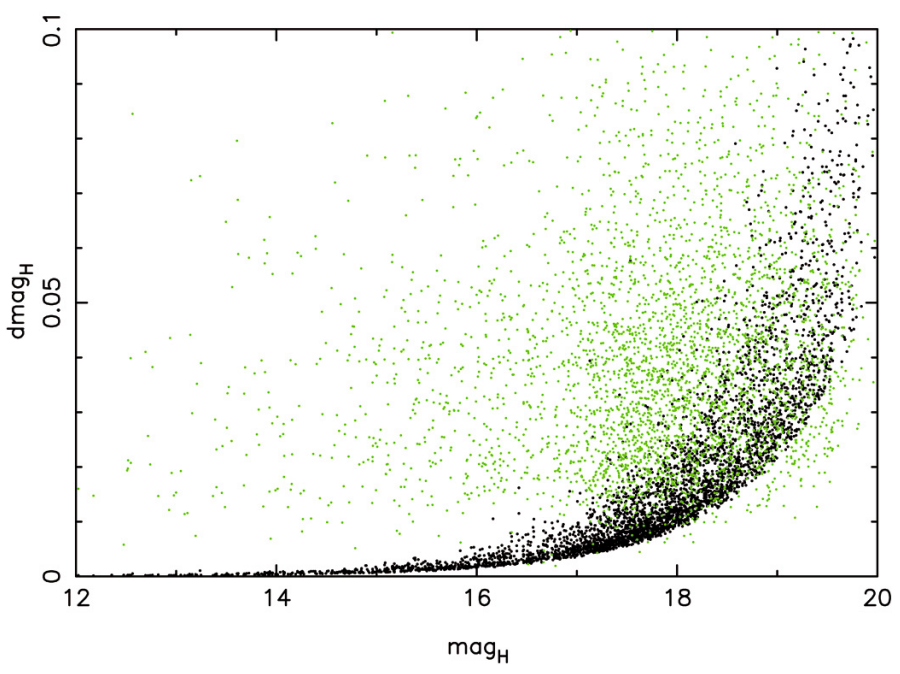

Fig. 3. Uncertainty in PSF fitting photometry with locally extracted PSFs in the image from dither position 3. Black dots are the formal photometric uncertainties computed by the StarFinder algorithm. Green dots are the photometric uncertainties due to the uncertainty in the PSF estimate.

\subsection{Deconvolution assisted local PSF fitting}

An astronomical image can be described by a convolution of the target ( $\delta$-functions in case of point-like sources) with the PSF:

$f(x, y)=o(x, y) \otimes p\left(x^{\prime}, y^{\prime}\right)$.

The target, $o(x, y)$, contains all the information about the observed object, while the PSF, $p\left(x^{\prime}, y\right)$, describes the imaging process (e.g., influence of atmosphere and telescope transfer functions). If the PSF is known, the object can be reconstructed by deconvolution techniques. Being an inverse process, deconvolution is, however, always an ill-posed problem, mainly because of limited knowledge of the PSF - due to the importance of noise and the limited coverage of spatial frequencies - and the presence of noise in the image. Deconvolution algorithms therefore have to use regularization techniques (e.g., Wiener filtering) to produce well-behaved solutions. For our work, we use and compare two common methods: (a) the Lucy-Richardson deconvolution, a maximum likelihood solution based on Bayes' theorem (Richardson 1972; Lucy 1974); and (b) linear Wiener filter deconvolution, where the image is divided by the PSF in Fourier space and a Wiener filter regularizes the solution (see, e.g., Starck \& Murtagh 2006 for a detailed description of the deconvolution problem and common methods).

We show a beam-restored (with a Gaussian beam of 3 pixel FWHM) Lucy-Richardson (LR) deconvolved image of the FOV from dither position 3 ( $H$-band) in the left panel of Fig. 6 and the same image deconvolved with a linear Wiener filter (LW) method in the right panel of this figure. The same PSF, extracted from IRS 7, was used in both cases (see inset in left panel of Fig. 2). I used the implementations of the LR algorithm from the astronomical image processing package dpuser, developed originally by (Eckart \& Duhoux 1991) and substantially extended and maintained by Thomas $\mathrm{Ott}^{4}$. The Wiener deconvolution was programmed using the IDL programming language.

\footnotetext{
${ }^{4}$ http://www.mpe.mpg.de/ ott/dpuser/index.html
} 

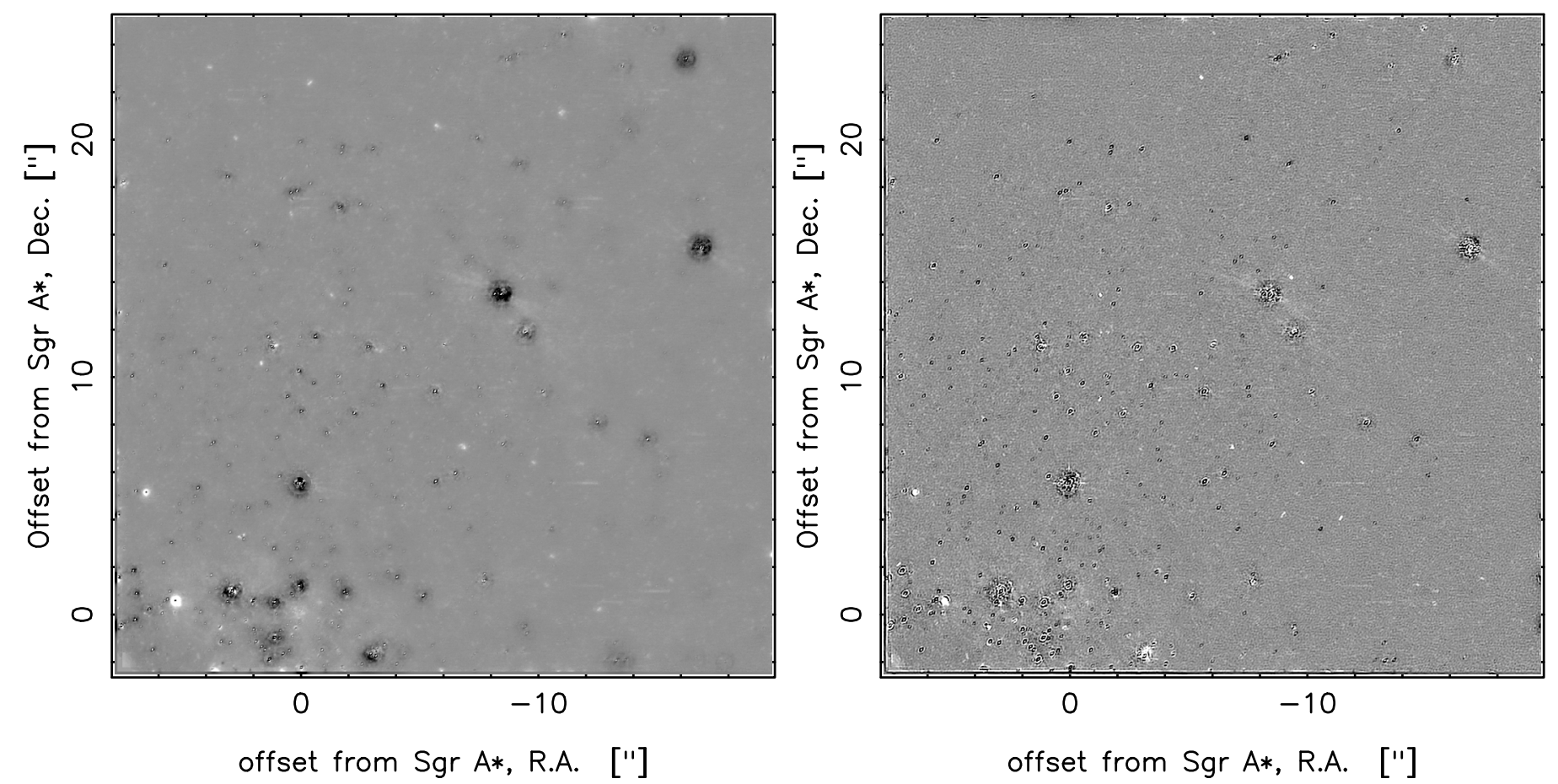

Fig. 4. Left: residuals (linear gray scale) after analyzing the image from dither position 3 with locally extracted PSFs. Right: residuals after LW deconvolution of the image from dither position 3 with the guide star PSF and local PSF fitting. The gray scale in both panels is identical to the one applied to the right panel of Fig. 2.
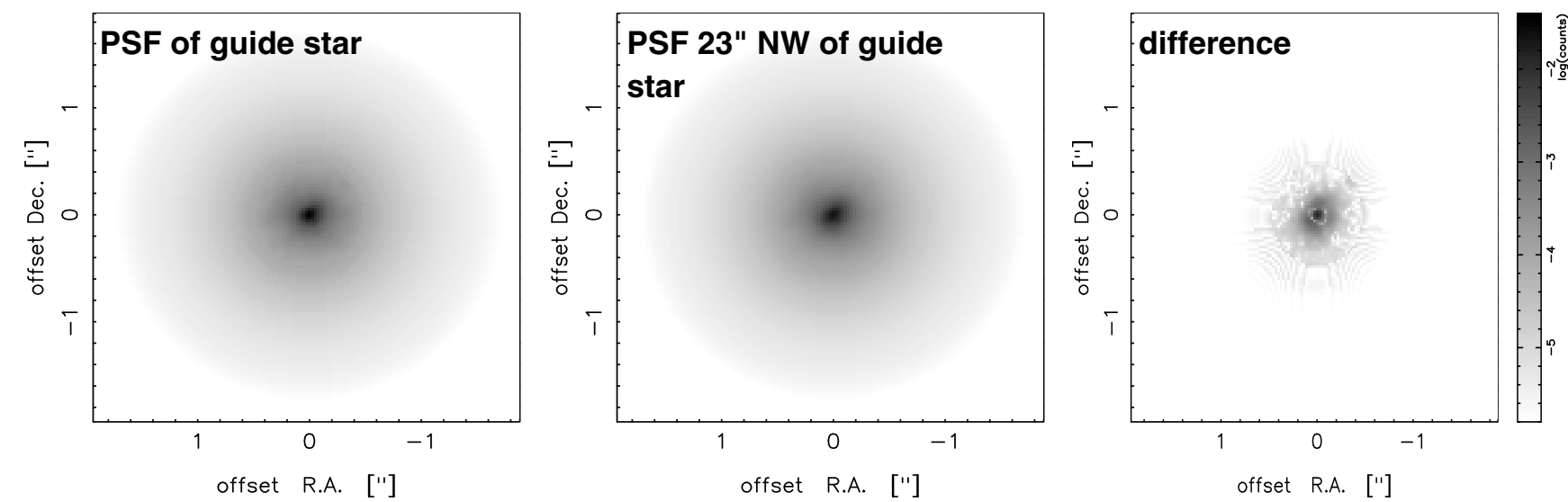

Fig. 5. Left: PSF at the position of the guide star. Middle: PSF $\sim 23^{\prime \prime} \mathrm{NW}$ of the guide star. Right: difference between PSFs in left and middle panels. The logarithmic gray scale is identical for all images and is indicated in the bar that accompanies the right panel. The circular feature that can be seen in the left and right panels is the radius beyond which the guide star PSF was radially and azimuthally smoothed.

In the presence of anisoplanatic effects, the PSF varies across the FOV. This can be described by a convolution with a spatially variable kernel:

$\operatorname{PSF}(x, y)=p(x, y) \otimes K\left(x^{\prime}, y^{\prime}\right)$,

where $p(x, y)$ is the PSF at the position of the guide star. Hence, when we deconvolve an AO image with the guide star PSF, we are left with $\delta$-functions at the positions of point sources in the ideal and isoplanatic case and with functions that describe the local kernel in the anisoplanatic case. Here, it must be noted that because of the discrete sampling of the image, a star is practically never described by a $\delta$-function. This would only be the case for a perfectly known PSF, at the location of the guide star, and if the stellar position happened to fall exactly onto the center of a pixel. Therefore, stars are always described by local kernels convolved with the PSF. Examples of local kernels are illustrated by the small insets in the left image in Fig. 6. If the guide star PSF has been used for deconvolution as in the example presented here, then the most compact kernels are found near the guide star, while the kernel is considerably more complex at distances larger than the isoplanatic angle.

How can deconvolution help to improve photometry in the presence of anisoplanatic effects? As we have seen, local PSF extraction is necessary in the presence of anisoplanacy. The main problem with local PSF extraction, however, occurs in obtaining accurate estimates of the wings of the PSFs. After deconvolution of the image with the guide star PSF, stars in the FOV appear in the shape of the local kernels at the corresponding positions. The sizes of these kernels are considerably smaller than the size of the original PSF. Hence, crowding is reduced. If we now perform the local PSF fitting algorithm, which was described in the preceding section, on the devonvolved image, the stars can be fit 


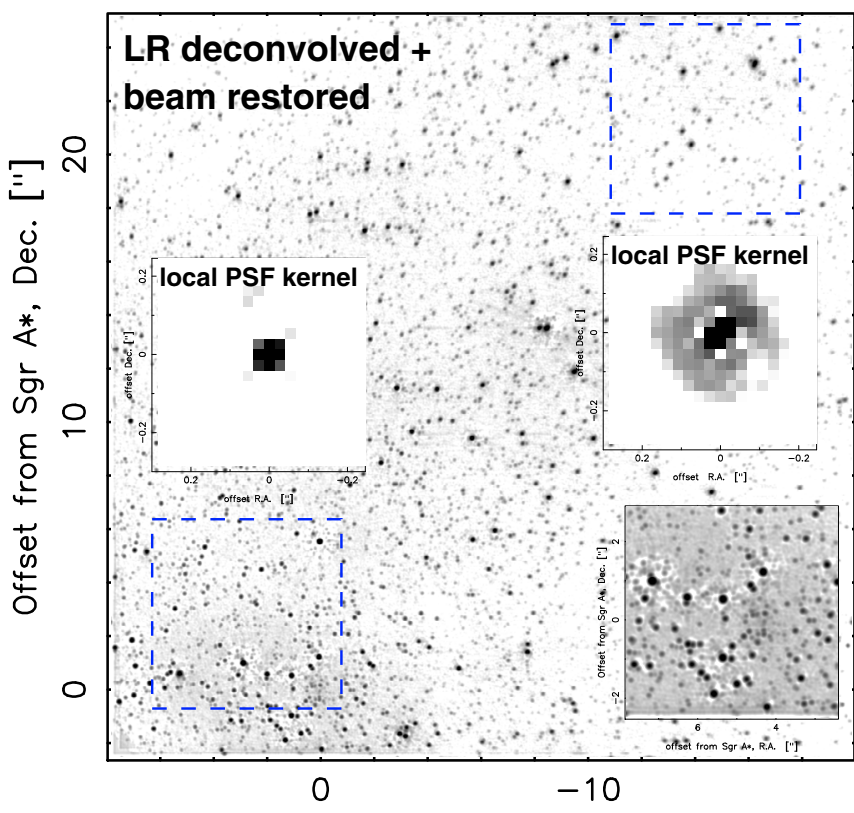

offset from Sgr A*, R.A. ["]

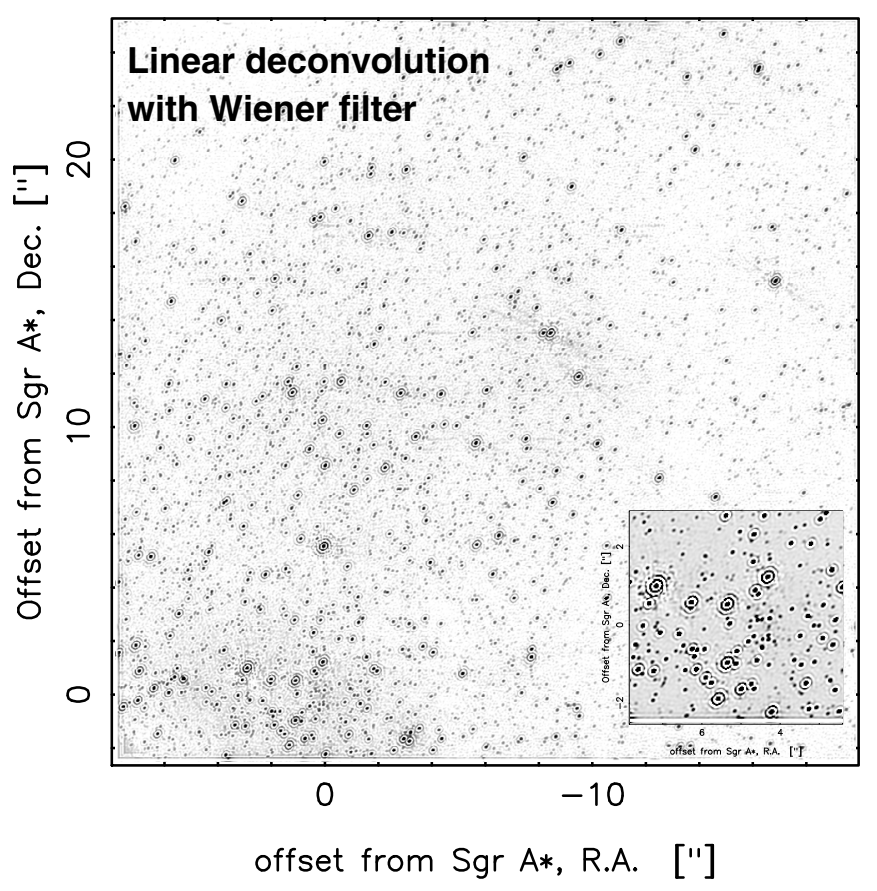

offset from Sgr A*, R.A. ["]

Fig. 6. Left: $H$-band image from dither position 3 after Lucy-Richard deconvolution and beam restoration with a beam of 3 pixel FWHM. Right: linear (Wiener filter) deconvolution of the same image. The insets in the left figure show the local PSF kernels extracted from 6.9" $\times 6.9^{\prime \prime}$ regions in two different fields of the LR deconvolved image (dashed boxes). The insets at the lower right corner of the two images show details near Sgr A*. Note that the typical ringing can be seen around the point sources in the Wiener-deconvolved image.

with the local kernels. Because of the reduced crowding and the increased $\mathrm{S} / \mathrm{N}$ of the point sources in the deconvolved image, the estimation of the local kernels can be achieved with high accuracy. In this way, we can realize local PSF fitting and circumvent the problem of having to truncate the locally extracted PSFs. This method will therefore lead to improved photometry of both the point sources and the diffuse background light.

The photometric uncertainties in the local PSF fitting photometry for the image of dither position 3 after prior LW deconvolution (using the guide star PSF) are shown in Fig. 7. When comparing with Fig. 3, one can see that the LW deconvolution prior to local PSF fitting leads to reduced scatter and generally lower PSF uncertainty. The formal uncertainties, on the other hand, appear to be slightly increased (they have been scaled by a factor of 3, see Sect. 4). The residuals related to point sources are not extended and appear to be homogeneous across the FOV (right panel of Fig. 4)

We note that deconvolution violates to a certain degree a basic assumption of PSF fitting, which is that the noise for each pixel is independent of that of adjacent pixels. Deconvolution will lead inevitably to covariances between the pixels. A variety of tests show that linear deconvolution does not lead to any significant bias, but care must be taken when assessing the uncertainties in the measured quantities adequately. This issue will be discussed in Sect. 5.1.

In the following subsection, we examine the effects of deconvolution by working on artificial images. We study the question of which deconvolution technique (LW or LR) is most closely suited to our purpose.

\section{Comparing methods on a simulated image}

In order to compare the performance of the various methods of photometry described above we created a simulated image. The anisoplanatic effect was modeled by using local PSFs to create

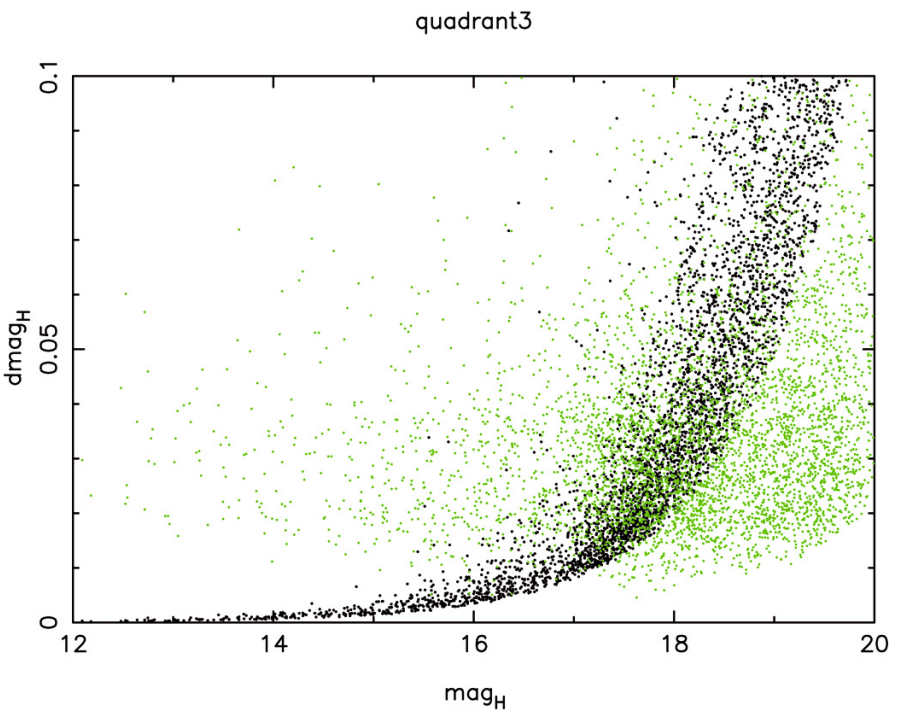

Fig. 7. Uncertainty in PSF fitting photometry applied to the image of dither position 3, like in Fig. 3. The difference here is that the image was deconvolved with the guide star PSF and the linear Wiener-filter algorithm prior to local PSF fitting.

the artificial image. For this purpose, the local kernels extracted from the LR deconvolved image from dither position 3 (see left panel in Fig. 6) were used. The kernels were extracted in a grid pattern from $256 \times 256$ pixel $^{2}$ subframes separated by steps of 128 pixels. An individual kernel was produced for each source by interpolating the kernels from the four grid points closest to the source, except for sources at the edge of the field, where the nearest kernel was used, without interpolation. Subsequently, each kernel was convolved with the PSF extracted from the guide star, IRS 7, and added to the artificial image at given positions and with a given flux. In this way, an image with a smoothly 

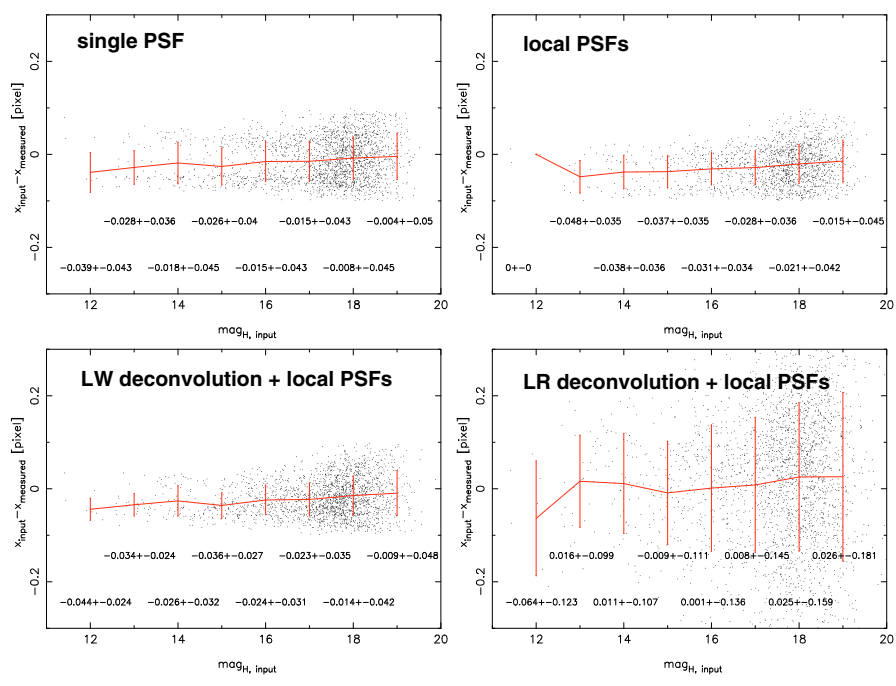

Fig. 8. Comparison of input and measured $\mathrm{x}$-axis positions in the simulated image. The labels give the mean differences and standard deviations (in pixels) per magnitude interval.

varying PSF was created ${ }^{5}$. The diffuse emission was set to a constant value. The fluxes and positions of the point sources were taken from the image of dither position 3. Gaussian readout noise and Poisson noise were added and the number of averaged exposures was chosen to coincide with the corresponding values of the data (see Table 1). PSF fitting photometry was performed in four different ways: (a) by extracting the PSF from the guide star, IRS 7, and fitting the entire image with this single PSF; (b) marking 200 reference stars over the entire FOV, local PSF fitting by creating local PSFs via extraction of PSF cores in overlapping subframes of size $\sim 6.9^{\prime \prime} \times 6.9^{\prime \prime}$, followed by merging with PSF wings from guide star, as described in section 3.2; (c) Wiener deconvolution with the PSF extracted from IRS 7 followed by local extraction and fitting of PSFs; (d) as (c), but using LR deconvolution.

The astrometry and photometry of the recovered point sources was finally compared with the input values. The extracted smooth diffuse background was compared to the input background (chosen to be a constant). The results are illustrated in Figs. 8-11.

\subsection{Astrometry}

The differences between input and recovered positions for the different methods are shown in Fig. 8 (only the $x$-axis values are shown, the $y$-axes values showing very similar behavior). LW deconvolution with subsequent local PSF fitting allows us to recover the positions of point sources with a standard deviation $<0.04$ pixels, except for the faintest sources. Local PSF fitting without deconvolution and fitting with a single PSF lead to results that are of similar quality. Lucy-Richardson deconvolution clearly deteriorates the astrometry, the standard deviations of the stellar positions being 2-3 times higher than in the other cases. We note that the mean of the differences between input and recovered positions is different from zero in all cases (by a few $1 / 100$ of a pixel). I propose that the most important factor for this behavior is that the position of a star has been defined

\footnotetext{
5 Note that the PSFs created in this way will be slightly broader than the PSFs in the original image because of the non-ideal properties of the kernels, as described in Sect.3.3 above. Broader PSFs will be a more challenging and therefore conservative way of testing the methods.
}
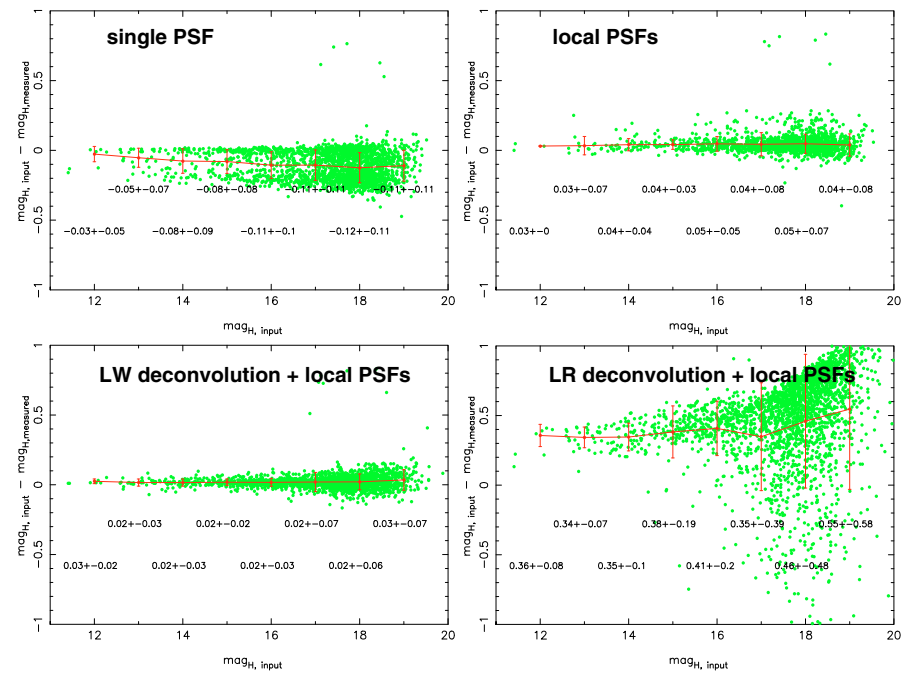

Fig. 9. Comparison of input and measured point source magnitudes in the simulated image. Green dots are the differences between input and output values (shifted along the $y$-axis). The labels give the mean differences and standard deviations per magnitude interval.

in the simulated images to coincide with the centroid position of the PSF (this is the usual standard for PSF fitting algorithms). In the artificial image, the stars are fixed at their locations and convolved with the complete PSF. StarFinder (like DAOPHOT) fits the positions and fluxes of the sources only within a fitting radius (or a fitting box in the case of StarFinder) and uses the complete PSF only for point source subtraction. The centroid of the PSF within the fitting radius (box) will differ from that of the entire PSF if the PSF outside the fitting radius is not pointsymmetrical. That is, the difference in mean positions between input and measurement is due to a difference between the PSFs used for input (entire model PSF) and output measurement (a partial PSF or a deconvolved PSF). In the case of a single PSF, it appears that there are actually two distributions of the differences between input and output, one centered on a slightly positive position, another one centered on slightly negative values. This is not necessarily surprising because the PSF becomes elongated with distance from the guide star, which may change the centroid position, depending on which side of the guide star a star is located. However, I did not fully explore this possibility because it would go far beyond the scope of this work if I were to explore the phenomenon of deviation between input and measured positions in detail here. However, I believe it is important to mention these points here because they may become of great significance in work that requires extremely precise astrometry in AO images. It may not be sufficient to determine stellar positions just from the PSF cores. At the moment, also I cannot exclude the possibility that positions may become biased by deconvolution, depending on the location in the field.

\subsection{Photometry}

The differences between input and recovered positions for the different methods are shown in Fig. 9. Both PSF fitting with a single PSF and PSF fitting after LR deconvolution produce significant deviations with the latter method providing the poorer results. An explanation of the bad performance of the LR algorithm can probably be found in its non-linearity. The LR deconvolution tends to be influenced by local noise peaks and incorporates the smooth background into the point sources. This leads 

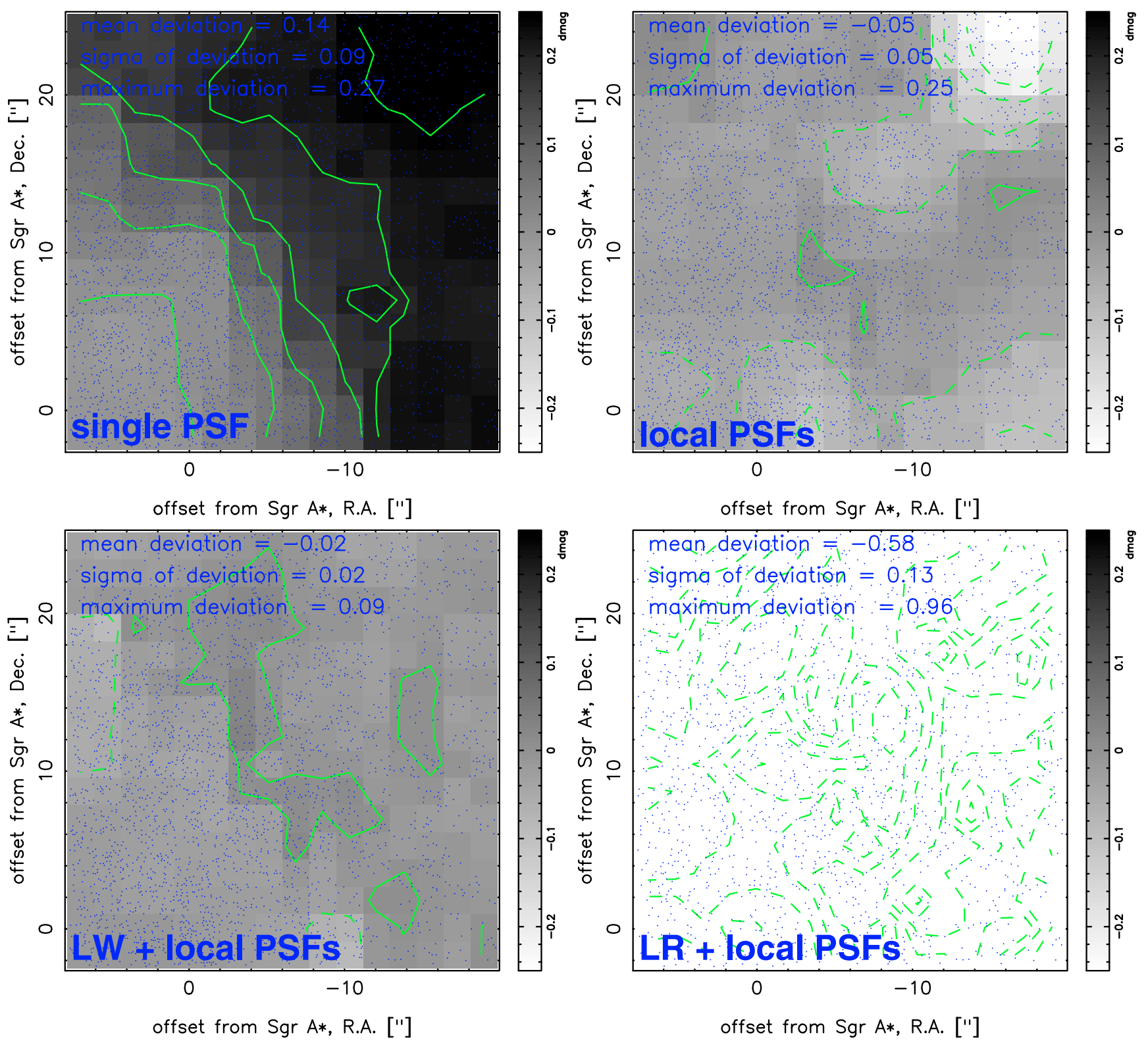

Fig. 10. Smoothed maps of the median differences (in boxes of $64 \times 64$ pixels) between the measured magnitudes of stars in a simulated image and the input magnitudes. The dots are the locations of detected stars. Upper left: PSF fitting with a single, fixed PSF extracted from the guide star. The contour lines indicate differences of 0.0-0.25 mag in steps of 0.05. Upper right: PSF fitting with locally extracted PSFs. Contour lines are plotted at 0.05 to $-0.2 \mathrm{mag}$ in steps of 0.05 . Lower left: PSF fitting with locally extracted PSFs after LW deconvolution with the guide star PSF. Contour lines are plotted at -0.05 (dashed), 0.0 , and 0.05 mag. Lower right: PSF fitting with locally extracted PSFs after LR deconvolution with the guide star PSF. Contour lines are plotted at -0.3 to -0.9 mag in steps of 0.1 . Note that in this case the panel is off the gray scale, which has been kept constant for all four panels.

to an increasing overestimation of the flux of faint sources and to characteristic empty patches with a size similar to the PSF around bright sources. Both local PSF fitting and local PSF fitting after linear deconvolution provide acceptable results. Local PSF fitting after Wiener deconvolution leads to the smallest standard and mean deviations. We note that the distribution for the single PSF (upper right panel in Fig. 9) appears bivariate. This may be related to the PSF properties discussed in the previous paragraph. Positive deviations, i.e., a source that is brighter in the measurement than in the input, are largely excluded in the case of a single PSF used for measurement. Elongation of the sources with distance from the guide star will lead to a loss of flux.
Smooth maps of the differences between input and measured photometry across the FOV are shown in Fig. 10. Using a single PSF leads to a systematic error that increases with distance from the guide star up to $\sim 0.25 \mathrm{mag}$. LR deconvolution combined with local PSF fitting does not lead to acceptable results, as we have already seen in Fig. 9. Local PSF fitting works quite well, only leading to systematic deviations in the upper right corner of the image, at extreme distances from the guide stars, where the PSFs are notably elongated. As an additional difficulty, there are no bright, isolated stars available in this region. Again, linear deconvolution followed by local PSF fitting is the most reliable method, leading to the smallest systematic variations across the FOV. 

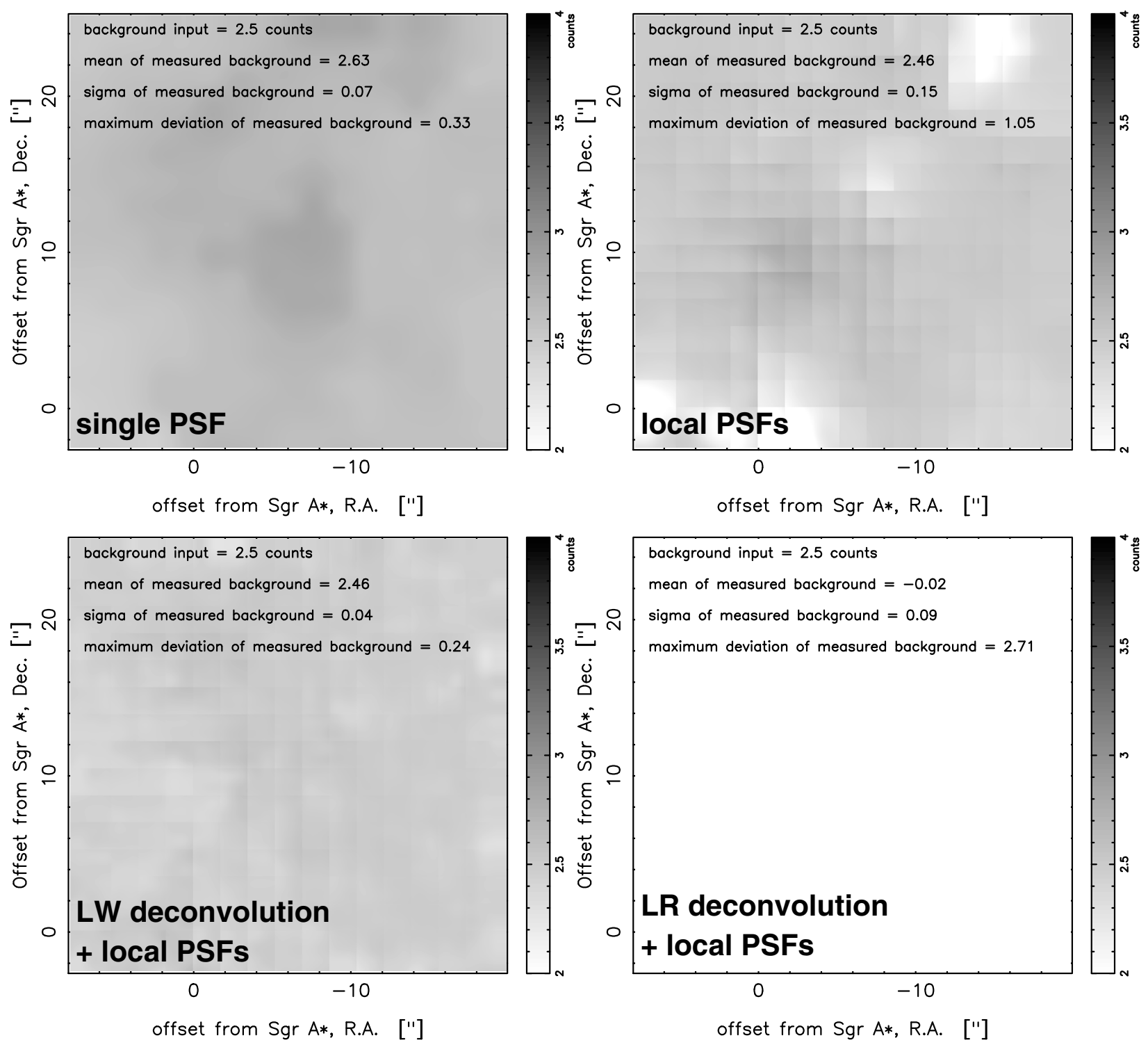

Fig. 11. Smooth background emission estimated for the point-source subtracted simulated image using different PSF fitting methods. Upper left: single, constant PSF. Upper right: local PSFs. Lower left: linear Wiener filter deconvolution and local PSF fitting. Lower right: Lucy-Richardson deconvolution and local PSF fitting. The gray scale is linear. Note the different gray scale in the lower right panel. The input background was constant and set to 2.5 counts (corresponding to a surface brightness of $15.5 \mathrm{mag} \operatorname{arcsec}^{-2}$ ). The labels give the mean difference, standard deviation, and maximum absolute deviation between measured and input background.

\subsection{Diffuse background}

The diffuse background emission in the artificial images was set to 2.5 counts, corresponding to $15.5 \mathrm{mag} \mathrm{arcsec}-2$. To ensure a reliable extraction of the background emission, it is again the Wiener deconvolution combined with local PSF fitting that produces the best results (Fig. 11). While single PSF fitting and local PSF fitting without deconvolution also produce acceptable results, they nevertheless cause significant systematic deviations across the FOV. LR deconvolution has the tendency to "scoop up" the diffuse emission into the point sources. The information on the background is largely lost in the LR deconvolved image. The smooth background emission is instead incorporated into the point sources. This is why the magnitudes of point sources in the LR deconvolved image become increasingly biased the weaker the sources are (see bottom right panel of Fig. 9) ${ }^{6}$.

\footnotetext{
6 This effect was taken care of in Buchholz et al. (2009), whose method assured accurate relative calibration of the point sources.
}

We conclude that the optimal method - among the ones that have been considered in this work - for extracting reliable photometry (and astrometry) for adaptive optics images with a FOV larger than the isoplanatic patch and sparsely sampled PSF is based on the following steps:

1. PSF extraction from the guide star (or from (a) bright star(s) near the guide star);

2. Wiener deconvolution of the image;

3. local PSF fitting.

We note that this method is not restricted to the case of the GC. It can probably be applied successfully in other cases where one is interested in accurate photometry over a large FOV in AO observations of crowded fields, but has to deal with sparse sampling of the PSF. 

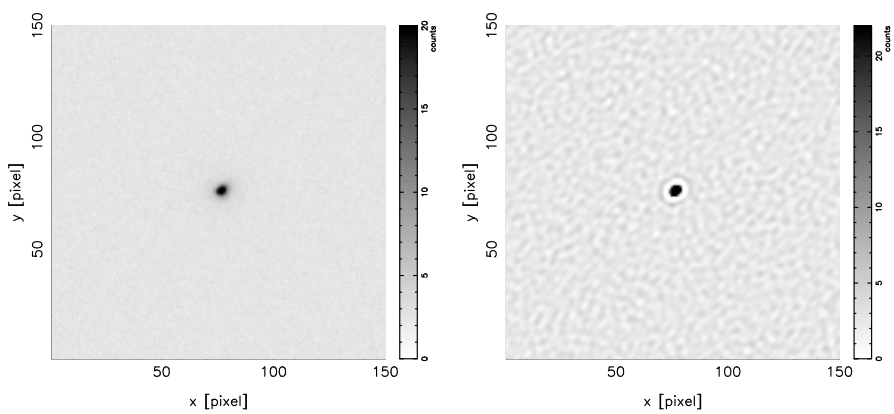

Fig. 12. Simulated image, using a background count of 2.5, readout noise of 4.2, electrons-per-ADU of 11.0, corresponding to the used NACO mode (224 exposures were used, i.e., $N=8$, NDIT $=28$ ). The single source at the center has 1000 counts. Left: raw image. Right: Wiener deconvolved image. The standard deviation of the raw sky is 0.34 counts, the one of the deconvolved sky 1.49 counts. The mean of the two skies is, of course, identical.

\section{Further tests}

\subsection{Deconvolution, noise, and uncertainties}

Deconvolution - at least in the linear case - can be regarded as re-imaging the data with a different set of (virtual) optics. However, the difference from real optics is that the true image has already been made and that the noise is already present in the data, the latter point explaining why deconvolution needs to be applied with some care. In this section, I discuss whether it is valid to combine deconvolution with PSF fitting techniques and what caveats have to be kept in mind.

PSF fitting is supposed to be applied to raw images, i.e., the noise statistics of the pixels should be preserved, which will be used to assign weights to the individual pixels (e.g., Stetson 1987). Deconvolution, even if it is linear as in Wiener deconvolution, will violate this assumption to a certain degree because it will lead to covariances between the pixels. This is illustrated in Fig. 12, which shows the raw and the deconvolved version of an artificial image of a sky (2.5 counts) containing 1 star (1000 counts): The deconvolved sky shows some "granularity" caused by the covariances between the pixels ${ }^{7}$. In a Monte Carlo simulation, 100 realizations of this image plus star were created. Subsequently, the position and flux of the star as measured in the raw and deconvolved images were compared. Table 2 gives the mean of the recovered position and flux as well as their corresponding standard deviations and the mean of the formal uncertainties estimated by StarFinder. The result of this simulation shows that the position and flux of the star are reliably recovered from the deconvolved image. However, the standard deviation of the measurements for the deconvolved image is somewhat larger than in the raw image (e.g., increase in flux uncertainty from $0.4 \%$ in the raw image to $0.7 \%$ in the deconvolved image) and the PSF fitting algorithm underestimates the true uncertainties of position and flux by a factor of $\sim 3.3$.

In a second simulation, 100 Monte Carlo simulations were run on the artificial star field used in Sect. 4. The local PSF fitting algorithm was performed on both the raw and the deconvolved images. In the case of the raw images, local PSFs were created

\footnotetext{
7 Note that in this work the Gaussian part of the noise in the data was estimated directly from the image and not from a number of exposures and readout noise. This is done by the StarFinder routine GAUSSIAN_NOISE_STD. Although this does not take care of the covariances, in this way it is at least possible to use a more conservative estimate of the noise due to Gaussian processes.
}

by merging locally extracted cores with the wings from the guide star PSFs. The guide star PSF was used for the deconvolution. Input and recovered positions and fluxes were compared to determine the true standard deviations of these quantities. Those were subsequently compared with the formal and PSF uncertainty estimates delivered by the PSF fitting algorithm. It can be seen in Fig. 13 that the scatter in the uncertainties is lower when Wiener deconvolution is applied. It can also be clearly seen that the formal uncertainties estimated by the PSF fitting algorithm (green stars) are underestimated in the deconvolved images. The correct uncertainties can however be reproduced when the formal uncertainties in the deconvolved images are scaled by a factor of $\sim 3$ before quadratically combining them with the PSF uncertainties. We note that the simulations may indicate that the PSF uncertainty is overestimated (at least for the bright stars). Since this is less of a problem than under-estimating the uncertainty, I have not investigated this point further for the time being.

In addition to these simulations, several other tests were performed to check whether the Wiener deconvolution introduces a significant bias in the astrometry or photometry of the data used in this work:

- as shown in Sect. 4, the simulated data (using numbers of exposures, read-out noise, gain corresponding to the data) show that Wiener deconvolution leads actually to the best astrometric and photometric results in the case of the presented data because it allows an accurate estimate of the local PSF;

- in Sect. 5.4, the uncertainties obtained from the photometry with Wiener deconvolution combined with local PSF fitting is checked by comparing results for sources present in the overlapping region of all four dither positions (see Sect. 3 and Fig. 1). This test suggests that the applied method delivers an accurate estimate of the photometric uncertainties;

- finally, Wiener deconvolution combined with local PSF fitting was applied separately to each of the 8 exposures for dither position 3. Mean positions and fluxes as well as the corresponding uncertainties of the sources present in all the exposures were derived from these independent measurements. The values were compared with the results obtained for the combined image. Again, the results coincide well within the uncertainties.

There appears to be a workaround to avoid performing PSF fitting on the deconvolved image. It consists of extracting local kernels from the deconvolved data and subsequently reconvolving them with the PSF that was used to deconvolve the entire image. The result would be a local PSF that could be applied to PSF fitting in the raw image to thus avoid the problem of altered noise statistics in the deconvolved image. Unfortunately, this approach does not lead to satisfactory results. Deconvolution - regardless of the actual method used - is an ill-posed problem and requires some kind of regularization. The purpose of the regularization is to suppress noise at high frequencies. This will lead inevitably to some loss of real information. In the case of Wiener deconvolution, the Wiener filter suppresses the highest frequencies. The trade-off is the characteristic ringing around point sources, or, if the reglarization parameter is large, to broad, Gaussian-like PSFs. The effect can be seen, e.g., in Fig. 12, where the PSF of the deconvolved source has a finite FWHM of a few pixels and is surrounded by a ring that is typical of Wiener deconvolution. Reconvolving the deconvolved source will therefore not recover the original image. The PSF in the reconvolved image will in fact be too broad. So, one cannot use the kernels extracted from deconvolved images to recover the PSF of the original image. 
Table 2. Results of Monte Carlo modeling of background plus one star (100 tries) as shown in Fig. 12.

\begin{tabular}{llllllllll}
\hline \hline & $x^{a}$ & $\sigma_{x, \text { formal }}^{b}$ & $\sigma_{x, \text { true }}^{c}$ & $y^{d}$ & $\sigma_{y, \text { formal }}^{\mathrm{e}}$ & $\sigma_{y, \text { true }}^{f}$ & $f^{g}$ & $\sigma_{f \text { formal }}^{h}$ & $\sigma_{f \text {,true }}^{i}$ \\
{$[\mathrm{pix}]$} & {$[\mathrm{pix}]$} & {$[\mathrm{pix}]$} & {$[\mathrm{pix}]$} & {$[\mathrm{pix}$ [pix] } & [counts] & [counts] & [counts] \\
\hline raw & 75.670 & 0.009 & 0.009 & 75.109 & 0.008 & 0.009 & 1000 & 4 & 4 \\
deconvolved & 75.670 & 0.003 & 0.011 & 75.108 & 0.003 & 0.010 & 1000 & 2 & 7 \\
\hline
\end{tabular}

${ }^{a}$ Measured position of the star on the $X$-axis (model position $x=75.670$ ) ${ }^{b} 1 \sigma$ uncertainty of measured $x$ given by the PSF fitting algorithm (average of the uncertainties of all the tries) ${ }^{c} 1 \sigma$ uncertainty of measured $x$ as given by the standard deviation of the individual measurements ${ }^{d}$ Measured position of the star on the $Y$-axis (model position $\left.x=75.110\right)^{e} 1 \sigma$ uncertainty of measured $y$ given by the PSF fitting algorithm (average of the uncertainties of all the tries) ${ }^{f} 1 \sigma$ uncertainty of measured $y$ as given by the standard deviation of the individual measurements ${ }^{g}$ Measured flux of the star (input flux $\left.f=1000.0\right)^{h} 1 \sigma$ uncertainty of measured $f$ given by the PSF fitting algorithm (average of the uncertainties of all the tries $)^{i} 1 \sigma$ uncertainty of measured $f$ as given by the standard deviation of the individual measurements.
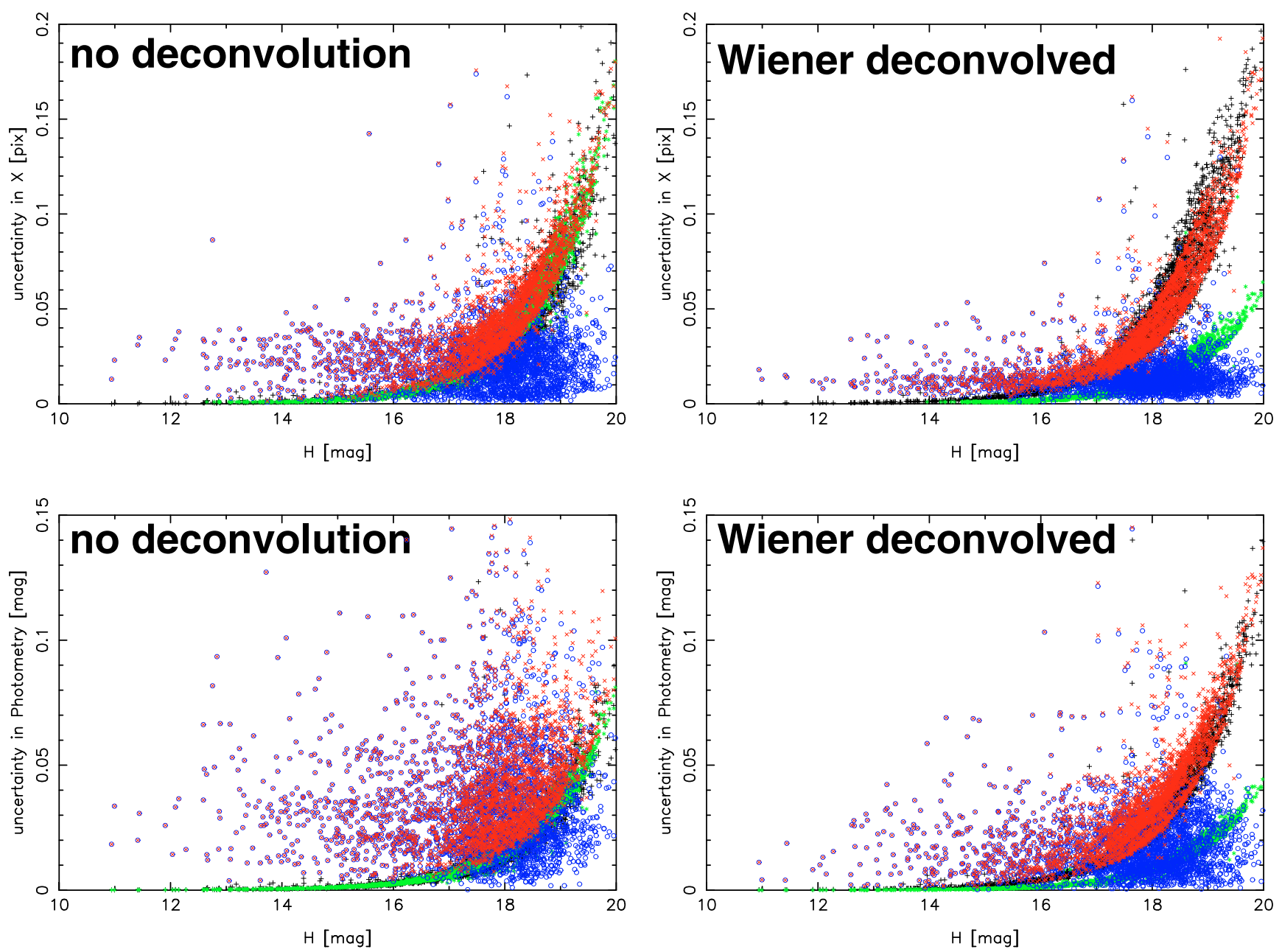

Fig. 13. Results of Monte Carlo simulations (100 runs) on an artificial image. Left: no deconvolution, PSF fitting after determining local PSFs via combination of local cores with the wings of the guide star PSF. Right: Wiener deconvolution before local PSF fitting. Upper panels: uncertainty in $X$-positions vs. magnitude. Lower panels: uncertainties in photometry vs. magnitude. Black plus signs (hardly visible in most parts, generally underlying the red crosses) are uncertainties determined from the standard deviations between input and measured positions and fluxes. Green stars are the formal uncertainties estimated by the PSF fitting algorithm. Blue rings are the PSF uncertainties. Red crosses are the combined formal plus PSF uncertainties. In the case of the Wiener deconvolved images, the formal uncertainties were scaled by a factor of 3 before combining them with the PSF uncertainties.

To conclude, I believe that the numerous tests applied to real and artificial data show that, although applying PSF fitting to a Wiener deconvolved image is not rigorously mathematically correct, the combination of Wiener deconvolution and PSF fitting works reliably and does not introduce any significant bias into the astrometry and photometry. But care must be taken to arrive at correct estimates of the uncertainties. The great advantage of (linearly) deconvolving the data is that one is then able to extract reliable local PSFs. However, there is a price that has to be paid. Deconvolution appears to increase the uncertainty in the measured positions and fluxes. Because of the covariances between the pixels that are caused by the deconvolution process, these uncertainties cannot be estimated directly from the PSF fitting algorithm. Instead, scaling factors must be applied to the formal uncertainties. The good news is that the scaling factor can be estimated in a fairly straightforward (but time-consuming) way by 
artificial star tests. Alternatively, multiple measurements of independent data sets can be combined to estimate the uncertainties and eliminate spurious sources.

\subsection{DAOPHOT}

The software package DAOPHOT, which is also included in the $I R A F$ program package, can be considered to represent the industrial standard on PSF fitting. It allows us to choose between six kinds of numerical PSFs, which are allowed to vary linearly or quadratically across the FOV. Additionally, a look-up table is produced to take into account the deviations of the true PSFs from the numerical model. Since a comparison with the standard is obviously important, I experimented on the data used in this paper with DAOPHOT, although, unfortunately, I was unable to produce any satisfying results. While I cannot exclude that this unsatisfying outcome is due to my lack of experience with DAOPHOT I nevertheless believe to have identified the following reasons for not having been successful:

- peculiar properties of low Strehl AO PSF. The FWHM of the guide star in the data used here is $\sim 3.5$ pixel or $0.095^{\prime \prime}$. The seeing foot of the complete PSF, however, has a radius of $\sim 60$ pixels or $1.62^{\prime \prime}$. This is more than a factor of 10 larger than the PSF core. This behavior is found in neither seeing-limited data nor data from HST, to which DAOPHOT is frequently applied. Additionally, there are just a handful of stars present in the image that are bright enough for the PSF to be traceable out to these large distances. For fainter stars, the PSF apparently disappears within the noise at radii $\sim 30-40$ pixels. Nevertheless, the combined action of the overlapping PSF wings of highly crowded bright stars, such as around Sagittarius A* (located at the origin in Fig. 1), makes it absolutely necessary to derive the full PSF out to 60 pixels. In the contrary case, one would also lose information about the diffuse emission de to unresolved stars. The latter carries valuable information about the structure of the Galactic center nuclear star cluster because it is currently possible to resolve only a few percent of the stars in this region (see Schödel et al. 2007);

- crowding of the field. Because of the large size of the PSF it is very difficult to find sufficiently bright and isolated stars as PSF references. IRS 7 is by far the most reliable PSF reference and in fact the only one that allows us to reliably estimate the PSF wings at large distances from the star;

- inhomogeneous distribution of stars. To derive a reasonable PSF model, it is important to have a sufficient number of isolated stars distributed roughly homogeneously across the FOV. This is clearly not the case here, where there are large patches without bright stars. Some of the bright sources in the field are also extended (bow-shocks and/or relation to diffuse emission in the close vicinity of the star). The lack of bright stars in some areas of the field, combined with the extended PSF wings, also appear to lead to systematic errors in the PSF look-up tables;

- naturally, I experimented with de-crowding the field with purely numerical or with only linearly variable PSFs. Unfortunately, this produced large residuals and did not lead to success. Possible reasons are that the available PSFs do not model the seeing foot of the PSF well enough in this case. An additional difficulty, which may only be true for the data used here is that even the core of the PSF is not symmetric (which is not rare in $\mathrm{AO}$ ). An indication of the inadequacy of the available mathematical PSF models may be that there was a strong degeneracy as to which mathematical model to apply for the PSF (basically all available functions gave very similar chi-squared values).

All these points can be briefly summarized in the statement that the PSF is complicated, very extended, and variable across the field, but there is only one good PSF reference star available (3 reference stars per degree of freedom are a minimum requirement for DAOPHOT and similar approaches). This lies at the root of this exercise. If the situation were easier, simple local PSF extraction with StarFinder would have produced satisfying results as well. I am confident that in this case DAOPHOT (or SExtractor) would have worked very well too. It is probably possible to modify the standard algorithms in ways that can deal with the described difficulties. These ways are indicated by the observation that the true seeing foot of the AO PSF does not vary drastically with position in the field. An accurate numerical description of the seeing foot combined with look-up tables for just the PSF cores may be an avenue worth exploring.

\subsection{Choice of initial PSF}

A source of uncertainty that has not yet been considered is the PSF used for the linear Wiener-filter deconvolution before the local point-source fitting. Because of the bright guide star, IRS 7, it is fairly easy to obtain an accurate estimate of the guide star PSF for the observations analyzed here. However, it is not obvious how to proceed in cases where there is no sufficiently bright and isolated star available for PSF extraction, as in a crowded field full of faint sources, or when the bright stars are saturated. Apart from these points, one must also keep in mind that the PSF used for deconvolution is always subject to uncertainties. How flexible is the presented method in terms of its constraints on the PSF used for the deconvolution?

Linear Wiener filter deconvolution is a linear process, i.e., it is reversible - with the caveat that some information is suppressed by the need to cut high frequencies with the Wiener filter. If there is a certain degree of inaccuracy in the PSF, this inaccuracy will be conserved in the local kernels after deconvolution. Since we fit the stars with the local kernels in the second step, any systematic uncertainty should be largely taken care of by the suggested method. We therefore expect no strong biases that may be introduced by an inaccurate PSF in the first step.

We performed tests of this hypothesis. The simulated image from Sect. 4 was LW deconvolved with different PSFs prior to local PSF fitting. We used (a) a broadened version of the guide star PSF (convolution of the guide star PSF with a 2 pixel Gaussian, i.e., s core of the PSF too broad); (b) a narrower version of the guide star PSF (obtained by raising the PSF to the power of 1.1 at all pixels); (c) a PSF derived from $9 \operatorname{mag}_{H} \approx 10$ stars within $5^{\prime \prime}$ of the guide star (i.e., under-estimation of the wings); and (d) a Moffat function that was fitted to the guidestar PSF (errors in both core and wings of the PSF). The systematic errors in the photometry of the point sources as a function of position in the FOV were limited to $\lesssim 0.03$ mag in all cases. Concerning deviations and uncertainties in photometry and astrometry, as well as the reliability to recover the diffuse background emission, the results are summarized in Table 3. The smallest errors correspond to when the errors of the PSF are confined to its core, while errors in the wings will have a more significant influence on the photometry, particularly on the reliability of the recovered diffuse emission. Not surprisingly, the pure mathematical model, the Moffat function, produces the poorest 
Table 3. Overview of consequences of errors in the estimated PSF.

\begin{tabular}{llllll}
\hline \hline & $\begin{array}{l}\Delta_{\text {mag }}^{e} \\
{[\mathrm{mag}]}\end{array}$ & $\begin{array}{l}\sigma_{\mathrm{mag}}^{f} \\
{[\mathrm{mag}]}\end{array}$ & $\begin{array}{l}\Delta_{\text {pos }}^{g} \\
{[\mathrm{pix}]}\end{array}$ & $\begin{array}{l}\sigma_{\text {pos }}^{h} \\
{[\mathrm{pix}]}\end{array}$ & $\begin{array}{l}\text { Diff } \\
\text { [counts] }, \sigma, \max \end{array}$ \\
\hline $\mathrm{a}^{a}$ & 0.02 & $0.02-0.11$ & 0.05 & $0.03-0.1$ & $2.45,0.50,0.4$ \\
$\mathrm{~b}^{b}$ & 0.03 & $0.01-0.13$ & 0.02 & $0.02-0.10$ & $2.70,0.26,1.9$ \\
$\mathrm{c}^{c}$ & 0.04 & $0.02-0.13$ & 0.05 & $0.025-0.10$ & $2.34,0.12,1.0$ \\
$\mathrm{~d}^{d}$ & 0.075 & $0.01-0.12$ & 0.03 & $0.016-0.10$ & $2.12,0.27,1.68$ \\
\hline
\end{tabular}

${ }^{a}$ PSF convolved with a 2 pixel FWHM Gaussian, i.e., too broad (mainly in the core). ${ }^{b}$ PSF with all pixels raised to the power of 1.1 , i.e., too narrow. ${ }^{c}$ PSF derived from several stars within $5^{\prime \prime}$ of the guide star, but $\sim 4$ mag fainter, i.e. PSF wings underestimated. ${ }^{d}$ Moffat function (fitted to guide star PSF) used as PSF. ${ }^{e}$ Approximate mean offset between input and measured magnitudes of the stars. ${ }^{f}$ Range (for magnitudes 12 to 19) of the standard deviation of the difference between input and measured magnitudes. ${ }^{g}$ Approximate mean offset between input and measured pixel positions of the stars (given for $X$, similar for $Y$ ). ${ }^{h}$ Range (for magnitudes 12 to 19) of the standard deviation of the difference between input and positions (given for $X$, similar for $Y$ ). ${ }^{i}$ Mean, sigma and maximum deviation of estimated diffuse background.

- but still satisfying - results (errors in both the core and wings of the PSF).

Of course, even when using IRS 7 as a PSF reference, there is a certain subjectivity in adjusting the smoothing parameters for the wings (the HALO_SMOOTH routine) and when choosing the final masking radius. However, several tests (varying the values of the smoothing parameters and masking radius by up to $20 \%$ ) show that the bias of these effects on the point source photometry is smaller than $1 \%$. It can, however, have an effect of order $10 \%-20 \%$ on the estimated diffuse emission near bright sources or in very densely populated areas.

We conclude that uncertainty in the PSF used for the primary deconvolution will lead to uncertainties of only a few percent in the measured flux of point sources, but can become of greater significance for the diffuse background. While the results show some robustness with respect to PSF errors, great care should be taken, nevertheless, when extracting the primary PSF, particularly its extended wings. This process is unfortunately never free of subjectivity and requires some experience. It is always recommendable to check the residuals and the diffuse flux related to the area of the image where the PSF reference star(s) is (are) located.

\subsection{Accuracy of the method tested on real data}

As an independent check of the photometric accuracy, we compared the photometric uncertainties derived from our algorithm (i.e., formal plus PSF uncertainties) with the uncertainties derived by comparing the measurement of stars present in all four dither positions of the $H$-band observations.

We extracted a PSF from the guide star, IRS 7, for each of the images corresponding to the four dither positions. The images were then deconvolved with the linear Wiener filter algorithm. Finally, local PSF fitting was performed on the deconvolved images. A scaling factor of 3.3 was applied to the formal uncertainties given by the PSF fitting algorithm to account for the under-estimation of uncertainties in deconvolved images (see Sect. 5.1). The lists of detected point sources in the overlapping subframes and the smooth background estimates and residuals for the overlapping subframes were combined as described in Sect. 3.2. Uncertainties were calculated by quadratically combining the formal fit uncertainties with the PSF uncertainties. For
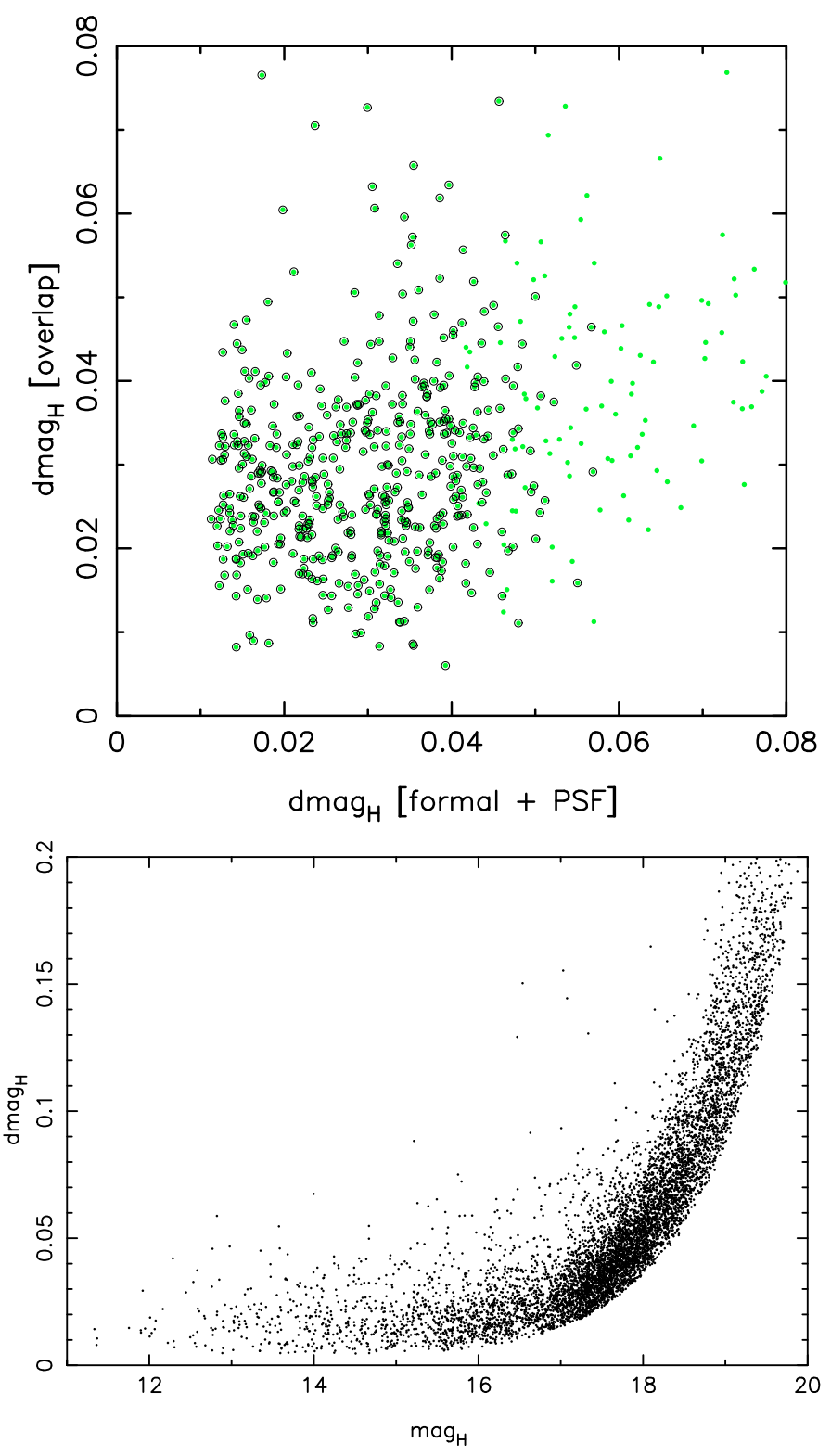

Fig. 14. Top: comparison between photometric uncertainties in the $H$-band measurements obtained from the algorithm applied here (scaled (see Sect. 4) formal fitting plus PSF uncertainties, $\operatorname{dmag}_{\text {formal+PSF }}$ ) and the uncertainties derived from stars in the field where the four dither positions overlap (dmag overlap ). Green dots: all stars in the common area; black circles: only stars with $\operatorname{mag}_{H} \leq 18$ Bottom: plot of photometric uncertainty vs. magnitude for all stars detected in the entire FOV (all four dither positions).

stars without multiple measurements. we adopted a PSF uncertainty of 0.02 mag (see Fig. 7).

The uncertainties derived from the algorithm (formal plus PSF uncertainties) are compared with those obtained from the four independent measurements in the overlapping fields in Fig. 14. The uncertainties appear to be uncorrelated and of similar magnitude. More than $91 \%$ (50\%) of the stars have a photometric uncertainty smaller than $0.05 \mathrm{mag}(0.03 \mathrm{mag})$. The bottom panel of Fig. 14 shows a plot of the photometric uncertainty versus magnitude for all stars detected in the combined (i.e., all four dither positions) FOV. To exclude spurious sources that may possibly originate in the deconvolved images, we excluded stars 

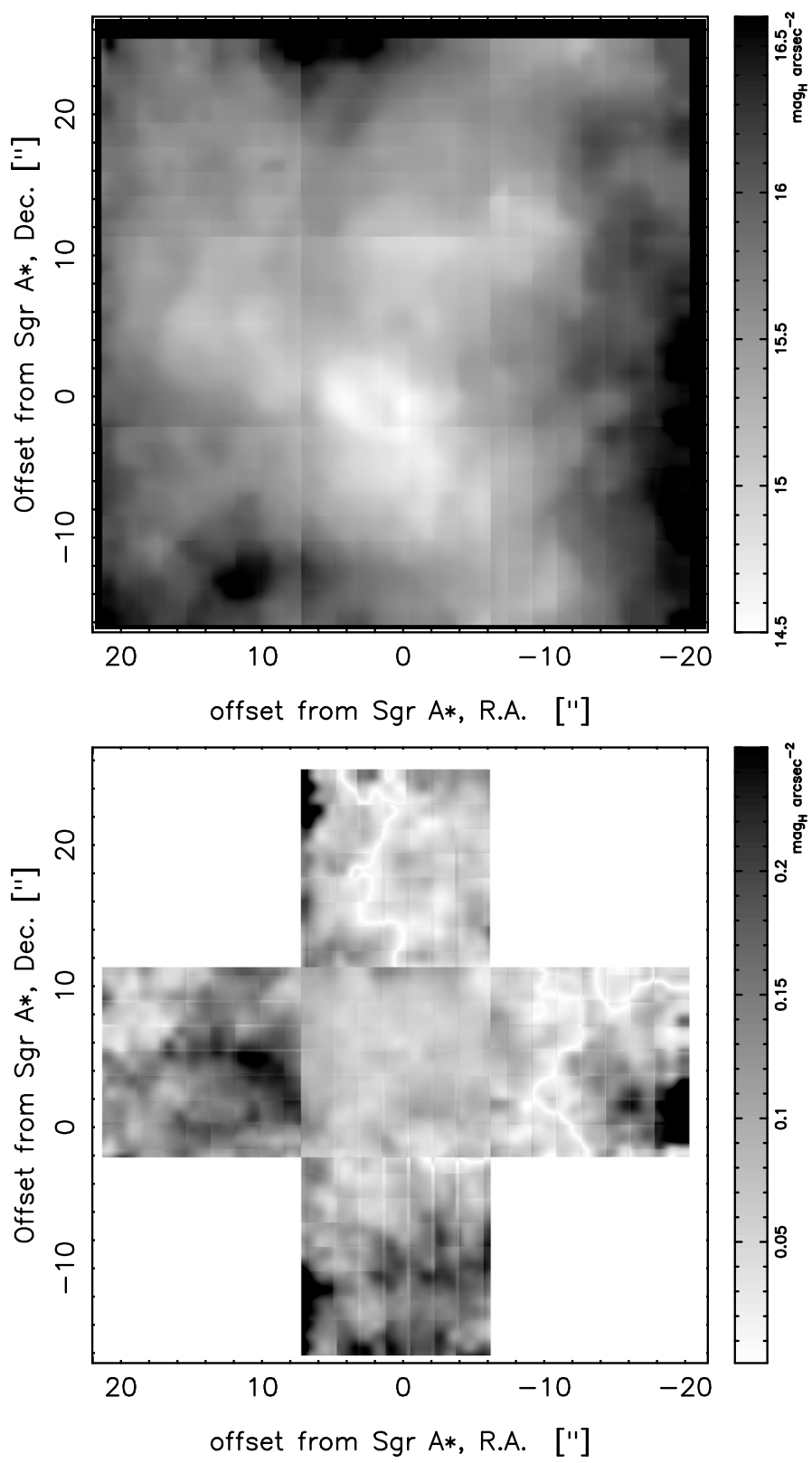

Fig. 15. Diffuse emission in the $H$-band FOV. The bottom panel illustrates the uncertainty in the diffuse emission, estimated from the overlap areas between the dither positions.

that are not also detected by local PSF fitting without prior deconvolution.

The diffuse emission extracted form the entire FOV of the $H$-band observations is shown in the top panel of Fig. 15. The checkerboard pattern is caused by our method because we have partitioned the field into many small overlapping sub-fields. The bottom panel of Fig. 15 shows the uncertainty in the measured diffuse background determined from the deviation between overlapping fields. It can again be seen that the applied algorithm appears to work very well. No systematic variations can be seen and the uncertainty is generally $\leq 0.1 \mathrm{mag} \operatorname{arcsec}^{-2}$, with the exception of some small patches, where the uncertainty can reach $\sim 0.25 \mathrm{mag} \operatorname{arcsec}^{-2}$.

\section{Summary and conclusions}

Photometry with PSF fitting algorithms depends critically on the ability to obtain accurate estimates of the PSF and its variability over the FOV. Standard software (DAOPHOT, SExtractor, StarFinder) can deal well with variable PSFs if there is a sufficient number of bright, unsaturated, and isolated stars present in the image. Additionally, these stars must be distributed in a roughly homogeneous way to adequately sample the PSF variability. In this work, we present a data set, an adaptive optics image of the Galactic center, that presents several particularities that make a standard approach difficult, leading to large systematic errors. The main difficulties are extremely extended PSF wings combined with strong crowding, significant variability of the PSF due to anisoplanatic effects, and, above all, a scarcity of adequate PSF reference stars in most parts of the fields. There is only a single excellent reference star present in the images. Local extraction of PSFs is limited in its accuracy by the variable density and brightness of the stars in the field, which leads generally to an underestimation of the extended wings of the PSFs and therefore an under-estimation of the brightness of point sources and a corresponding overestimation of the diffuse emission.

This work shows that in such a difficult case one can obtain accurate photometric and astrometric results using two methods: (a) assuming that the wings of the PSFs, i.e., the seeing foot of the AO PSF, do not vary significantly across the FOV. In that case, local PSFs can be estimated by combining locally extracted PSF cores with the wings of the PSF of a bright star (e.g., the guide star); (b) using the PSF from one or several suitable bright star(s) (e.g., the guide star) for Wiener deconvolution followed by PSF fitting with locally extracted PSFs. Both methods lead to satisfying results. The method involving Wiener deconvolution is superior. There is a caveat to take into account, however. Deconvolution will alter the noise statistics of the images. In this work, several tests and simulations have shown that this will not lead to any systematic bias in the astrometry or photometry, but to an underestimation of the uncertainties in the PSF fitting algorithm. This can be taken into account by either comparing measurements of several independent data sets or by determining a scaling factor for the uncertainties from Monte Carlo simulations.

The method involving Wiener deconvolution is fairly tolerant to the PSF used in this primary deconvolution, but it is ideally extracted from (a) bright star(s) close to the guide star. In the case of the Galactic center and for the data shown here, this approach works successfully. Systematic deviations of brightness across the field have a standard deviation of $\sim 0.02 \mathrm{mag}$. This should be compared with the photometric bias that varies systematically across the FOV reaching values as high as $\sim 0.2$ mag that occurs when using a single PSF for the entire $28^{\prime \prime} \times 28^{\prime \prime}$ FOV (Fig. 10). This corresponds to an improvement in accuracy by an order of magnitude. The presented method allows one additionally to estimate the diffuse emission due to non-resolved sources in a crowded field with an accuracy of $\sim 10 \%$.

The method was tested on simulated images. The diffuse emission can be recovered with high accuracy, i.e., deviations of $<10 \%$. Of course, this latter number also depends on the resolution with which the diffuse emission is estimated. Here we have used a box size of $30 \times 30$ pixels, i.e., about 10 times the FWHM of the PSF or $0.8^{\prime \prime} \times 0.8^{\prime \prime}$. Deviations in the estimated diffuse emission can become larger than $10 \%$ when the PSF used for primary deconvolution is not determined carefully.

An apparent disadvantage of linear Wiener filter deconvolution is the typical ringing produced around point sources. 
However, this is no problem when a PSF fitting algorithm is applied to the deconvolved image because the rings around point sources are considered features of the PSF. Since the Wiener filter deconvolution is a linear algorithm, information does not become destroyed as in the case of non-linear methods such as the Lucy-Richardson algorithm, which leads to deteriorated astrometry and photometry and loss of the information contained in the diffuse background.

Finally, we emphasize that our experiments have shown that it is important to take into account the photometric uncertainty introduced by our limited knowledge of the PSF. While the formal uncertainty in the point source astrometry and photometry can become arbitrarily small with the increasing brightness of the source (excluding saturation), there is a principal limit imposed by the accuracy with which the PSF can be determined. In the case analyzed here, for example, the minimum photometric uncertainty due to the PSF may be of the order 0.01-0.02 mag. The minimum PSF uncertainty depends on the details of the observations, particularly on the availability of bright, non-saturated PSF reference stars and on the level of crowding. We note that the PSF is not constant even in the case of a FOV smaller than the isoplanatic angle. Therefore, for high precision astrometry and photometry, the position dependence of the PSF should always be taken into account when the FOV is not significantly smaller than the isoplanatic angle.

This work does not intend to provide a readily available standard solution for PSF fitting with a spatially variable PSF. As has been mentioned before, convenient program packages already exist for these cases. The intention of this work was instead to determine ways in which one can deal with extremely difficult situations, when the standard methods may not work satisfyingly. My hope is that this work may also inspire new approaches to the problem in general of variable PSF fitting. Further improvement is probably possible by implementing the true local PSF estimation in StarFinder and, possibly, by taking covariances between the pixels into account. More work on the statistical effects of (Wiener) deconvolution on astronomical images could also lead to further progress.

The method for obtaining accurate photometry over a large FOV in AO images with a sparsely sampled PSF, as suggested in this paper, has been applied to NACO $H$-, $K \mathrm{~s}^{-}$, and $L p$-band imaging data of the Galactic center. The results (photometry of point sources and of diffuse emission) will be presented and discussed in an upcoming paper (Schoedel et al., submitted to A\&A).

Acknowledgements. R.S. acknowledges the Ramón y Cajal programme of the Spanish Ministerio de Ciencia e Innovación. R.S. thanks the anonymous reference for his help in improving this work.

\section{References}

Beckers, J. M. 1988, in ESO Conference on Very Large Telescopes and their Instrumentation, 2, 703

Bertin, E., \& Arnouts, S. 1996, A\&AS, 117, 393

Blum, R. D., Sellgren, K., \& Depoy, D. L. 1996, ApJ, 470, 864

Bouy, H., Kolb, J., Marchetti, E., et al. 2008, A\&A, 477, 681

Britton, M. C. 2006, PASP, 118, 885

Buchholz, R. M., Schödel, R., \& Eckart, A. 2009, A\&A, 499, 483

Christou, J. C., Pugliese, G., Köhler, R., \& Drummond, J. D. 2004, PASP, 116, 734

Devillard, N. 1997, The Messenger, 87, 19

Diolaiti, E., Bendinelli, O., Bonaccini, D., et al. 2000a, A\&AS, 147, 335

Diolaiti, E., Bendinelli, O., Bonaccini, D., et al. 2000b, ed. P. L. Wizinowich, SPIE Conf. Ser., 4007, 879

Eckart, A., \& Duhoux, P. R. M. 1991, ed. R. Elston, ASP Conf. Ser., 14, 336

Ellerbroek, B., Britton, M., Dekany, R., et al. 2005, ed. R. K. Tyson, \& M. Lloyd-Hart, SPIE Conf. Ser., 5903, 20

Gullieuszik, M., Greggio, L., Held, E. V., et al. 2008, A\&A, 483, L5

Hammer, F., Puech, M., Assemat, F. F., et al. 2004, ed. A. L. Ardeberg, \& T. Andersen, SPIE Conf. Ser., 5382, 727

Lucy, L. B. 1974, AJ, 79, 745

Marchetti, E., Brast, R., Delabre, B., et al. 2008, SPIE Conf. Ser., 7015

Ott, T., Eckart, A., \& Genzel, R. 1999, ApJ, 523, 248

Pott, J.-U., Eckart, A., Glindemann, A., et al. 2008, A\&A, 487, 413

Richardson, W. H. 1972, J. Opt. Soc. Amer. (1917-1983), 62, 55

Schödel, R., Eckart, A., Alexander, T., et al. 2007, A\&A, 469, 125

Schödel, R., Merritt, D., \& Eckart, A. 2009, A\&A, 502, 91

Starck, J.-L. \& Murtagh, F. 2006, Astronomical Image and Data Analysis, ed. J.-L. Starck, \& F. Murtagh (Berlin Heidelberg: Springer-Verlag)

Steinbring, E., Faber, S. M., Hinkley, S., et al. 2002, PASP, 114, 1267

Stetson, P. B. 1987, PASP, 99, 191

Stetson, P. B. 1992, in Astronomical Data Analysis Software and Systems I, ed. D. M. Worrall, C. Biemesderfer, \& J. Barnes, ASP Conf. Ser., 25, 297 